\title{
Angewandte
}

Supporting Information

\section{Impact of Organic Templates on the Selective Formation of Zeolite Oligomers}

Marine Ciantar, Thuat T. Trinh, Carine Michel, Philippe Sautet, Caroline Mellot-Draznieks, * and Carlos Nieto-Draghi*

anie_202014027_sm_miscellaneous_information.pdf 


\section{Supporting information}

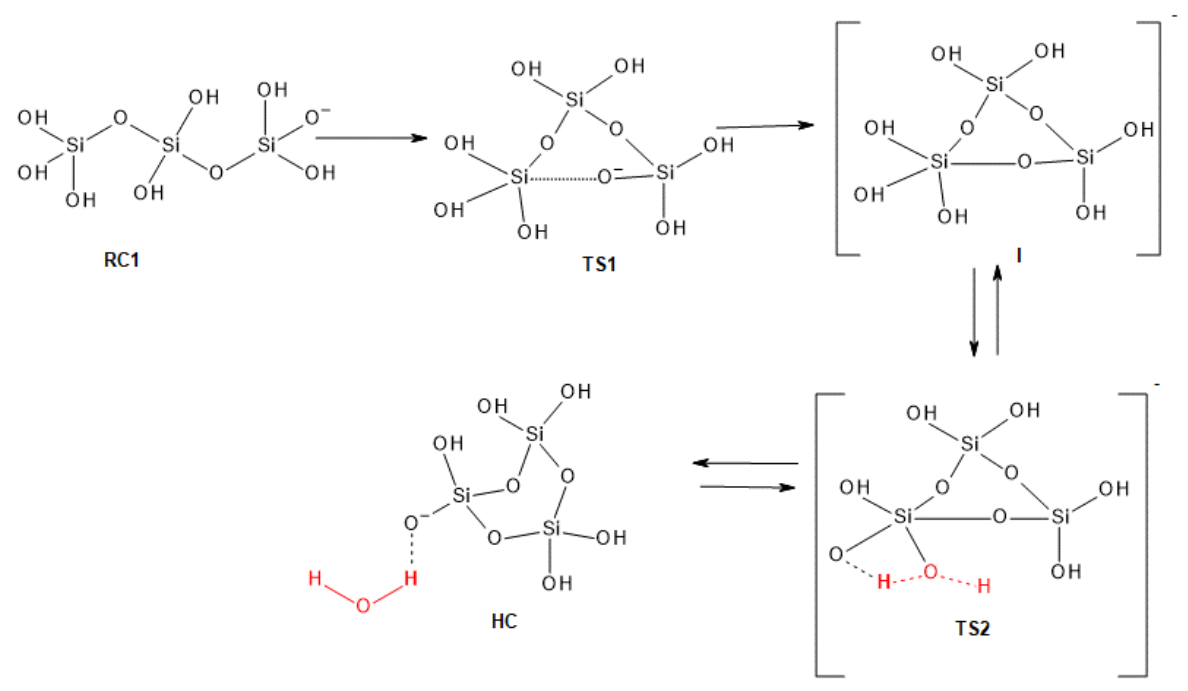

Scheme S1. Anionic concerted mechanism for the formation of cyclic oligomers. This mechanism involves a first reactive complex (RC1) formed from anionic, followed by a transition state (TS1) and a five-coordinated intermediate (I). A second transition state (TS2) allows the formation of a hydrated complex (HC).
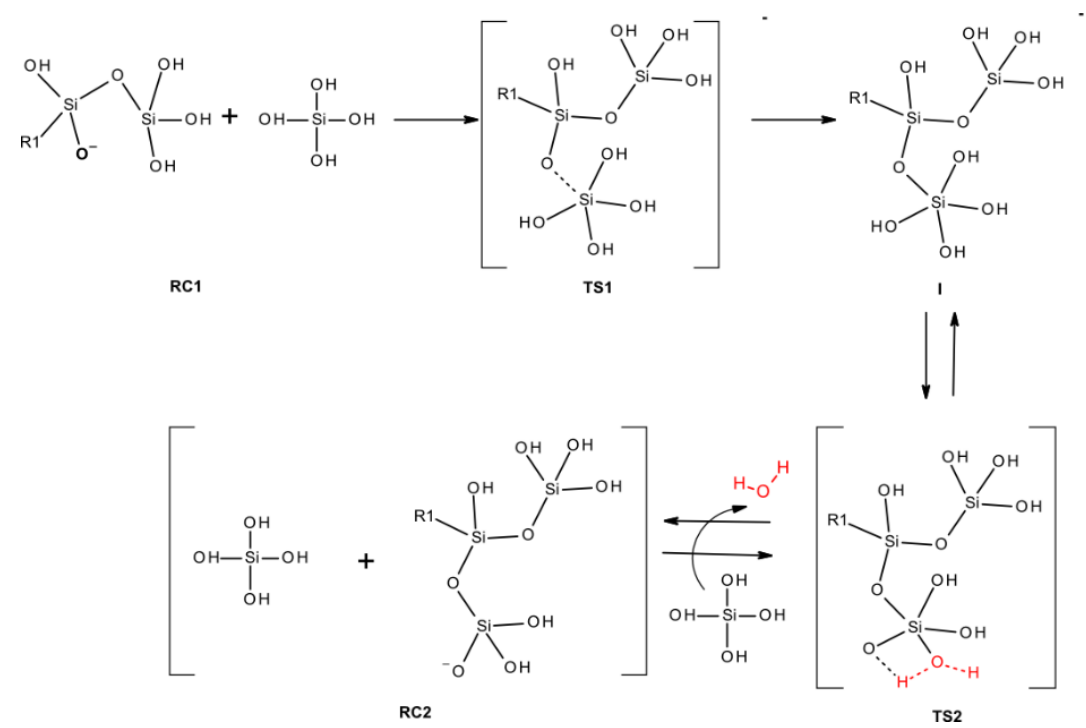

Scheme S2. Anionic concerted mechanism for the formation of branched oligomers. This new mechanism involves a first reactive complex (RC1) formed from anionic and neutral species, followed by a transition state (TS1) and a five-coordinated intermediate (I). A second transition state (TS2) allows the formation of a hydrated complex $(\mathrm{HC})$. A neutral monomer combines with $\mathrm{HC}$ to form a reactive complex (RC2) with the release of a water molecule. 


\section{SI Figures:}

\section{$\mathrm{NCl}$ Analysis of the role of templates in the intramolecular $\mathrm{H}$-bonds of pentacoordinated}

intermediates:

Further understanding of how the template influences the key pentacoordinated intermediate $\mathbf{I}_{\mathbf{i}}$ (Scheme 1 in main text) can be provided using a Non-Covalent Interaction analysis $(\mathrm{NCl})[1,2]$. This is based on the electron density and the reduced density gradient and allow the identification of the specific interactions between the template and the pentacoordinated intermediate $\mathbf{I}_{\mathbf{i}}$. The branched intermediate is stabilized by two intramolecular $\mathrm{H}$-bonds either in the absence or presence of a template (indicated by arrows in Figures S1.b and S1.c, respectively), pointing towards a minor role of TPA ${ }^{+}$in this case. By contrast, $\mathrm{TMA}^{+}$allows the formation of two additional intramolecular $\mathrm{H}$-bonds upon the pentamer's cyclisation (Figure S1.C, right) to complement the two H-bonds formed in absence of a template (Figure S1.b right)

(a) Free energy profiles
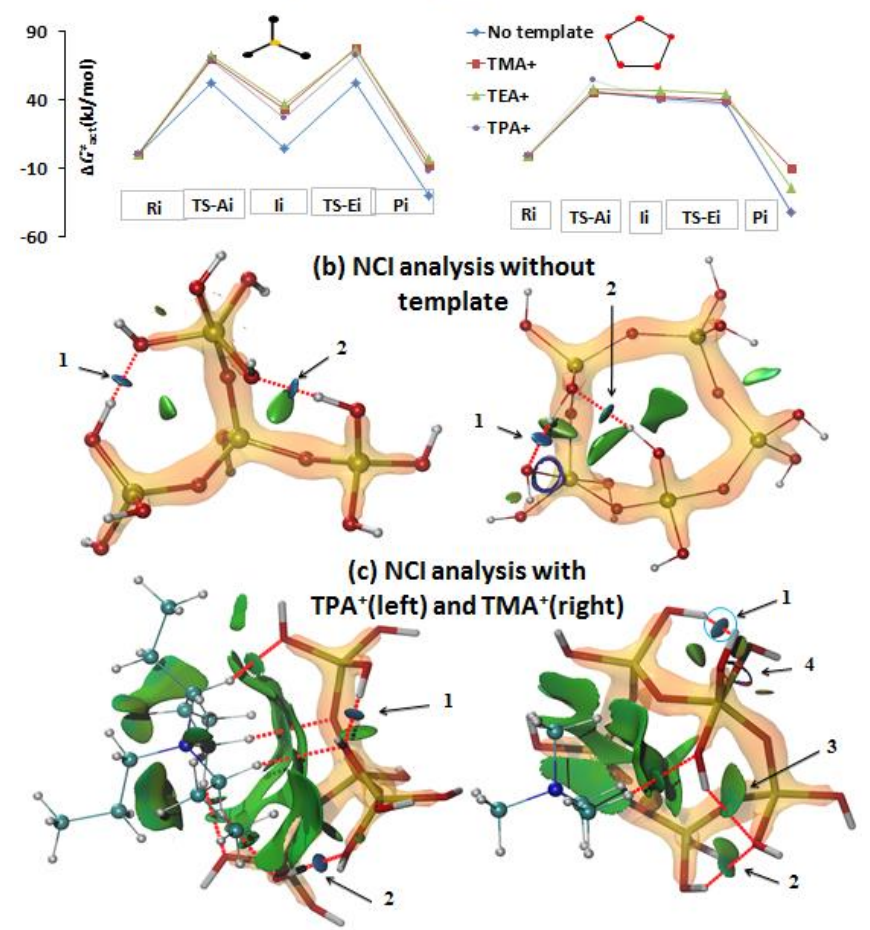

Figure S1. Comparison of branched tetramer (left) and cyclic pentamer (right). a) Free energy profiles from reactive complex $\left(\mathbf{R}_{\mathrm{i}}\right)$ to hydrated complex $\left(\mathbf{P}_{\mathrm{i}}\right)$ obtained with and without templates. $\mathrm{NCl}$ analysis for the conformation of oligomers without (b) and with (c) TPA+ or TMA+ template showing the intramolecular H-bonds (highlighted by arrows 1 to 4). Colours in figures (b) and (c) represent the different types of non-covalent interactions: H-bonds (blue); van der Waals (green). 

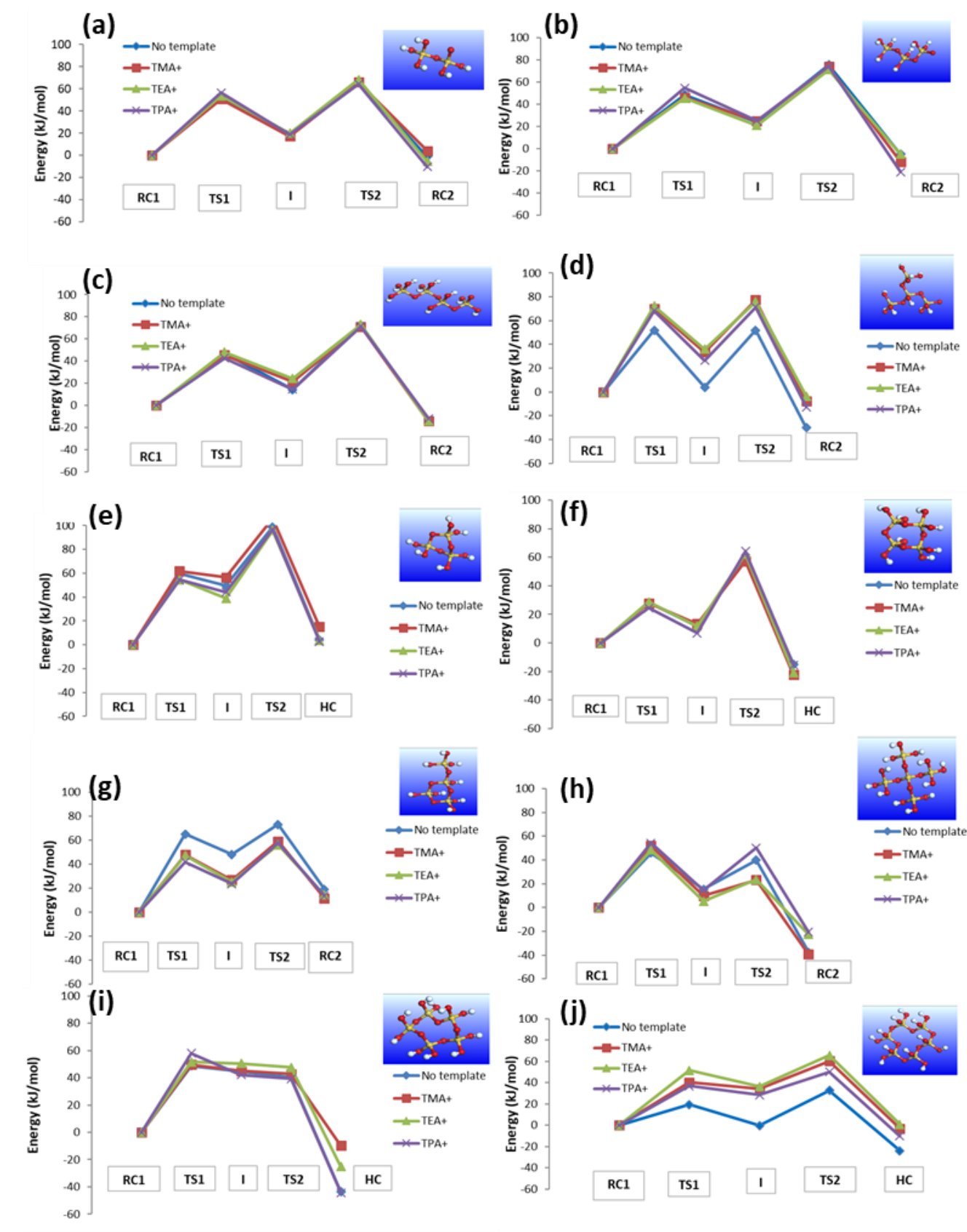

Figure S2: Comparison of the DFT activation barriers for different species with and without templates $\left(\mathrm{TMA}^{+}, \mathrm{TPA}^{+}\right.$and $\left.\mathrm{TEA}^{+}\right)$. 


\begin{tabular}{|c|c|c|c|c|c|}
\hline \multirow[b]{2}{*}{ Branched pentamer } & $\Delta \mathrm{G}$ & $\mathrm{KJ} / \mathrm{mol}$ & \multirow[b]{2}{*}{ TMA+ } & \multirow[b]{2}{*}{ TEA+ } & \multirow[b]{2}{*}{ TPA+ } \\
\hline & States & $\sqrt{\text { No template (SA) }}$ & & & \\
\hline \multirow[b]{6}{*}{ Cyclic Pentamer (5-ring) } & RC1 & 0 & 0 & 0 & 0 \\
\hline & |TS1 & 46 & 52 & 48 & 54 \\
\hline & | & 15 & 10 & 5 & 15 \\
\hline & |TS2 & 40 & 23 & 23 & 50 \\
\hline & RC2 & -37 & -39 & -23 & -21 \\
\hline & RC1 & 0 & 0 & 0 & 0 \\
\hline \multirow[b]{5}{*}{ Cyclic Hexamer (6-ring) } & |TS1 & 49 & 50 & 52 & 58 \\
\hline & 1 & 45 & 45 & 51 & 42 \\
\hline & TS2 & 40 & 43 & 48 & 39 \\
\hline & $\mathrm{HC}$ & -44 & -10 & -25 & -44 \\
\hline & $\overline{R C 1}$ & 0 & 0 & 0 & 0 \\
\hline \multirow{4}{*}{ 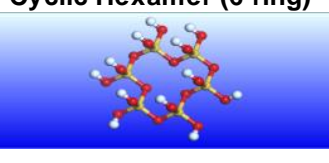 } & |TS1 & 19 & 40 & 52 & 37 \\
\hline & 1 & 0 & 34 & 36 & 28 \\
\hline & TS2 & 33 & 60 & 65 & 50 \\
\hline & $\mathrm{HC}$ & -24 & -3 & 1 & -10 \\
\hline \multirow{5}{*}{ Cvclic Tetramer (4-rina) } & RC1 & 0 & 0 & 0 & 0 \\
\hline & |TS1 & 28 & 28 & 29 & 25 \\
\hline & | & 12 & 13 & 12 & 7 \\
\hline & TS2 & 60 & 57 & 62 & 64 \\
\hline & HC & -15 & -22 & -21 & -15 \\
\hline \multirow[t]{2}{*}{ Cyclic Trimer (3-ring) } & $\mathrm{RC1}$ & 0 & 0 & 0 & 0 \\
\hline & TS1 & 60 & 62 & 55 & 55 \\
\hline \multirow[b]{4}{*}{ Branched tetramer } & I & 49 & 57 & 39 & 44 \\
\hline & TS2 & 99 & 106 & 96 & 97 \\
\hline & $\mathrm{HC}$ & 4 & 15 & 4 & 3 \\
\hline & $\mathrm{RC1}$ & 0 & 0 & 0 & 0 \\
\hline \multirow{4}{*}{$\therefore \sum_{0}^{\infty}$} & |TS1 & 52 & 70 & 72 & 68 \\
\hline & 1 & 4 & 33 & 36 & 27 \\
\hline & |TS2 & 52 & 77 & 76 & 71 \\
\hline & $\mathrm{RC}_{2}$ & -30 & -8 & -4 & -13 \\
\hline \multirow{5}{*}{$\begin{array}{c}\text { Branched 3-rina } \\
\\
9\end{array}$} & $\mathrm{RC1}$ & 0 & 0 & 0 & 0 \\
\hline & TS1 & 65 & 48 & 47 & 42 \\
\hline & 1 & 48 & 27 & 25 & 24 \\
\hline & TS2 & 73 & 59 & 56 & 58 \\
\hline & $\mathrm{RC} 2$ & 19 & 12 & 14 & 14 \\
\hline \multirow[t]{5}{*}{ Dimer } & RC1 & 0 & 0 & 0 & 0 \\
\hline & |TS1 & 53 & 51 & 54 & 57 \\
\hline & 1 & 18 & 17 & 20 & 19 \\
\hline & |TS2 & 65 & 66 & 68 & 64 \\
\hline & RC2 & -1 & 4 & -6 & -10 \\
\hline \multirow[t]{5}{*}{ Trimer } & $\mathrm{RC1}$ & 0 & 0 & 0 & 0 \\
\hline & |TS1 & 49 & 46 & 46 & 55 \\
\hline & I & 24 & 25 & 21 & 25 \\
\hline & |TS2 & 75 & 74 & 71 & 74 \\
\hline & $\mathrm{RC2}$ & -5 & -12 & -5 & -21 \\
\hline \multirow[t]{5}{*}{ Tetramer } & $\mathrm{RC} 1$ & 0 & 0 & 0 & 0 \\
\hline & |TS1 & 47 & 45 & 48 & 43 \\
\hline & | & 14 & 22 & 24 & 15 \\
\hline & |TS2 & 73 & 71 & 73 & 71 \\
\hline & $\mathrm{RC}_{2}$ & -14 & -14 & -14 & -12 \\
\hline
\end{tabular}

Table S1: Energies $(\mathrm{kJ} / \mathrm{mol})$ for oligomers until $\mathrm{Si}^{6}$ with all the intermediates species (RC1: reactive complex, TS1: transition state 1, I: Intermediate state, TS2: transition state 2, RC2 or HC: product complex or hydrated complex). 
(a)

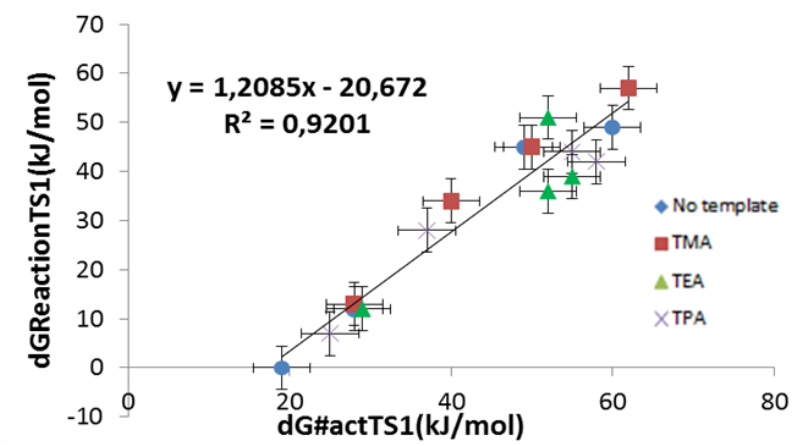

(b)

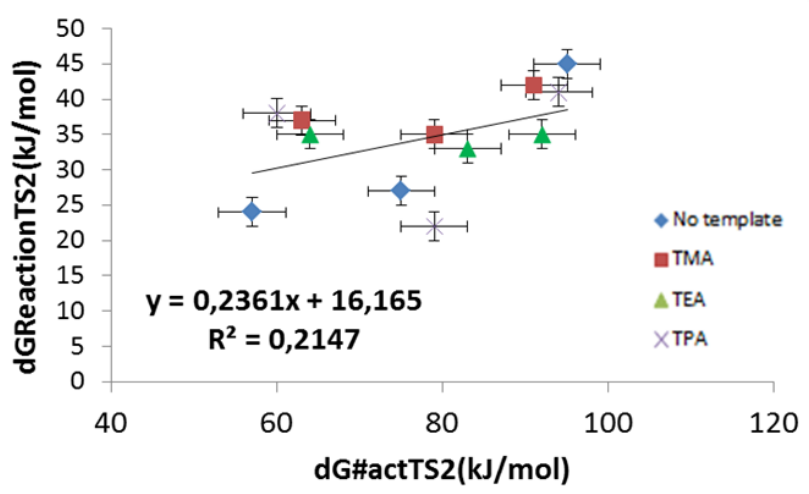

Figure S3: Brønsted-Evans-Polanyi relationship observed between the reactant complex RC1 and the intermediate I (a) and between I and RC2 (b) for cyclic oligomers as obtained from DFT calculations.
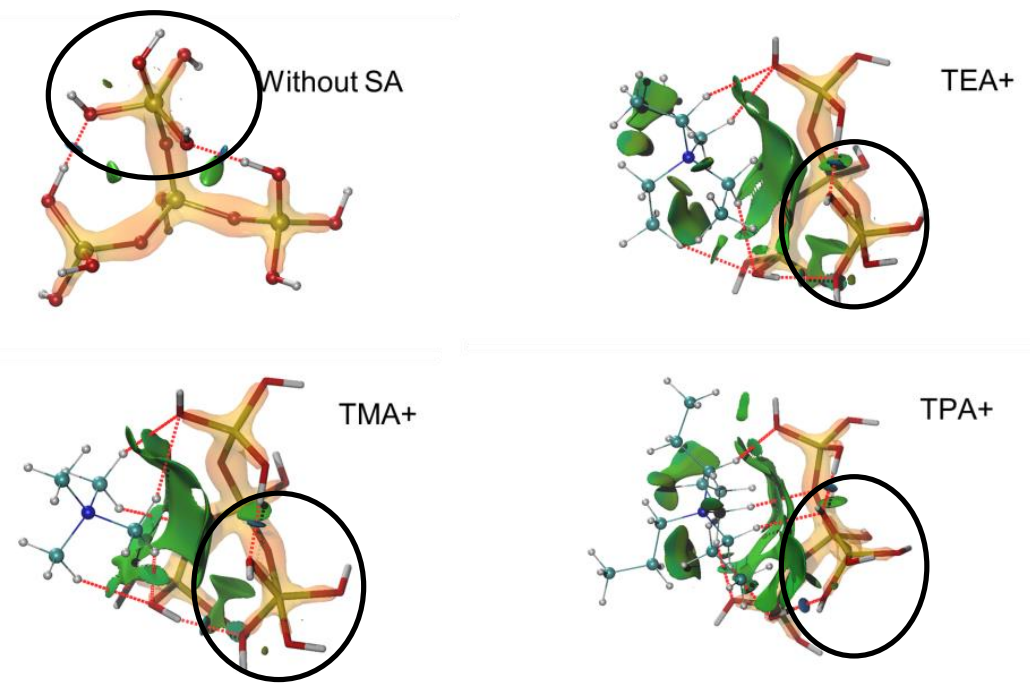

Figure S4: $\mathrm{NCl}$ Analysis for branched tetramer (Intermediate state) on the S2 scheme without and with templates (TEA+, TMA+ and TPA+). Colours in figures (b) and (c) represents different type of non-covalent interactions: $\mathrm{H}$-bonds (blue); vdW (green). Template-oligomer vdW interactions (in green) produce a conformation (black circle) with a lot of intermolecular bonds on the pentacoordinated silicium, which disfavors the release of the water molecule required for the formation of the product structure. 


\begin{tabular}{|c|c|c|c|c|c|c|c|c|c|c|c|c|c|}
\hline Number & \multicolumn{2}{|c|}{ No template } & \multicolumn{2}{|c|}{ TMAt } & \multicolumn{2}{|c|}{ TEAt } & \multicolumn{2}{|c|}{ TPAt } & \multicolumn{3}{|c|}{ Reaction details } & \multirow{2}{*}{$\frac{\text { Species }}{\text { ESP1 }}$} & \multirow{2}{*}{$\begin{array}{c}\text { Formula } \\
\mathrm{SI}(\mathrm{OH}) 4 \ldots, \mathrm{Si}(\mathrm{OH}) 3 \mathrm{O}-\end{array}$} \\
\hline 1 & 0 & 28 & 0 & 28 & 0 & 28 & 0 & 28 & esp22+esp23 & $\leftrightarrow$ & esp1 & & \\
\hline 2 & 53 & 35 & 50 & 34 & 49 & 36 & 50 & 36 & \multirow{2}{*}{$\begin{array}{l}\text { esp1 } \\
\text { esp2 }\end{array}$} & $\leftrightarrow$ & esp2 & \multirow{2}{*}{$\begin{array}{l}\text { ESP2 } \\
\text { ESP3 }\end{array}$} & [Si(OH)4-O-Si(OH)3]- \\
\hline 3 & 47 & 66 & 52 & 65 & 53 & 71 & 49 & 71 & & $\leftrightarrow$ & esp 3 & & $\mathrm{Si2O} \mathrm{OH}-{ }_{-,}, \mathrm{H} 2 \mathrm{O}$ \\
\hline 4 & 0 & 30 & 0 & 34 & 0 & 19 & 0 & 20 & \multirow{3}{*}{$\begin{array}{l}\text { esp3+esp22 } \\
\text { esp4 } \\
\text { esp5 }\end{array}$} & $\leftrightarrow$ & esp4+esp24 & \multirow{3}{*}{$\begin{array}{l}\text { ESP4 } \\
\text { ESP5 } \\
\text { ESP6 }\end{array}$} & $\mathrm{Si}(\mathrm{OH}) 4, \ldots \mathrm{Si} 2 \mathrm{O} 6 \mathrm{H} 5$ \\
\hline 5 & 49 & 25 & 46 & 21 & 46 & 25 & 55 & 30 & & $\leftrightarrow$ & esp5 & & [Si(OH)4-O-Si2O6H5]- \\
\hline 6 & 51 & 80 & 49 & 86 & 50 & 76 & 49 & 95 & & $\leftrightarrow$ & esp 6 & & $\mathrm{Si} 3 \mathrm{O} 10 \mathrm{H} 7-\ldots, \mathrm{H} 2 \mathrm{O}$ \\
\hline 7 & 0 & 29 & 0 & 35 & 0 & 40 & 0 & 21 & \multirow{3}{*}{$\begin{array}{l}\text { esp6 } \\
\text { esp7 } \\
\text { esp8 } \\
\end{array}$} & $\leftrightarrow$ & esp7+esp24 & \multirow{3}{*}{$\begin{array}{l}\text { ESP7 } \\
\text { ESP8 } \\
\text { ESP9 }\end{array}$} & Si309H7-O-( $(\Delta)$ \\
\hline 8 & 60 & 11 & 62 & 5 & 55 & 16 & 55 & 11 & & $\leftrightarrow$ & esp8 & & 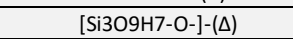 \\
\hline 9 & 50 & 95 & 49 & 91 & 57 & 92 & 53 & 94 & & $\leftrightarrow$ & esp9 & & 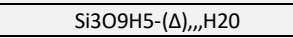 \\
\hline 10 & 0 & 21 & 0 & 14 & 0 & 30 & 0 & 10 & \multirow{3}{*}{$\begin{array}{c}\text { esp6+esp22 } \\
\text { esp10 } \\
\text { esp11 }\end{array}$} & $\leftrightarrow$ & esp10+esp24 & \multirow{3}{*}{$\begin{array}{l}\text { ESP10 } \\
\text { ESP11 } \\
\text { ESP12 }\end{array}$} & $\mathrm{Si}(\mathrm{OH}) 4, \ldots \mathrm{Si} 3 \mathrm{O} 10 \mathrm{H} 8$ \\
\hline 11 & 47 & 33 & 45 & 23 & 48 & 24 & 43 & 28 & & $\leftrightarrow$ & esp11 & & [Si(OH)3-O-Si3O1OH8]- \\
\hline 12 & 59 & 87 & 49 & 85 & 49 & 87 & 56 & 83 & & $\leftrightarrow$ & esp12 & & $\mathrm{Si} 4 \mathrm{O} 13 \mathrm{H} 9-,, \mathrm{H} 2 \mathrm{O}$ \\
\hline 13 & 0 & 30 & 0 & 50 & 0 & 66 & 0 & 44 & \multirow{3}{*}{$\begin{array}{c}\text { esp6+esp22 } \\
\text { esp13 } \\
\text { esp } 14\end{array}$} & $\leftrightarrow$ & esp13+esp24 & \multirow{3}{*}{$\begin{array}{l}\text { ESP13 } \\
\text { ESP14 } \\
\text { ESP15 }\end{array}$} & $\mathrm{Si}(\mathrm{OH}) 4, \ldots \mathrm{Si} 3 \mathrm{O} 10 \mathrm{H} 8(+)$ \\
\hline 14 & 52 & 47 & 70 & 37 & 72 & 36 & 68 & 41 & & $\leftrightarrow$ & esp14 & & {$[\mathrm{Si}(\mathrm{OH}) 3-\mathrm{O}-\mathrm{Si} 3 \mathrm{O} 10 \mathrm{H} 8]-(+)$} \\
\hline 15 & 48 & 82 & 44 & 85 & 40 & 80 & 44 & 84 & & $\leftrightarrow$ & esp15 & & Si4013H9-(+) ${ }_{1, \mathrm{H} 2 \mathrm{O}}$ \\
\hline 16 & 0 & 13 & 0 & 18 & 0 & 20 & 0 & 21 & \multirow{3}{*}{$\begin{array}{l}\text { esp12 } \\
\text { esp16 } \\
\text { esp17 }\end{array}$} & $\leftrightarrow$ & esp16+esp24 & ESP16 & Si4012H9-O-(0) \\
\hline 17 & 28 & 16 & 28 & 15 & 29 & 17 & 25 & 18 & & $\leftrightarrow$ & esp17 & ESP17 & {$[\mathrm{Si} 4012 \mathrm{Hg}-\mathrm{O}-]-(0)$} \\
\hline 18 & 48 & 75 & 44 & 79 & 50 & 83 & 57 & 79 & & $\leftrightarrow$ & esp18 & ESP18 & $\mathrm{Si} 4 \mathrm{OO} 2 \mathrm{H} 7-(0)_{1,1, \mathrm{H} 2 \mathrm{O}}$ \\
\hline 19 & 0 & 33 & 0 & 16 & 0 & 17 & 0 & 23 & esp12 & $\leftrightarrow$ & esp19+esp24 & $\overline{\text { ESP19 }}$ & Si4012H9-O-(0-branched) \\
\hline 20 & 65 & 17 & 48 & 21 & 47 & 22 & 42 & 18 & esp19 & $\leftrightarrow$ & esp20 & ESP20 & [Si4012H9-O-]-( $(0$ branched) \\
\hline 21 & 25 & 54 & 32 & 47 & 31 & 42 & 34 & 44 & esp20 & $\leftrightarrow$ & esp21 & ESP21 & $\mathrm{Si} 4012 \mathrm{H} 7-(0 \text { branched })_{,}, \mathrm{H} 2 \mathrm{O}$ \\
\hline 22 & 0 & 21 & 0 & 14 & 0 & 30 & 0 & 10 & esp $12+$ esp 22 & $\leftrightarrow$ & esp28 + esp24 & ESP22 & $\mathrm{Si}(\mathrm{OH}) 4$ \\
\hline 23 & 47 & 33 & 45 & 23 & 48 & 24 & 43 & 28 & esp28 & $\leftrightarrow$ & esp29 & ESP23 & $\mathrm{Si}(\mathrm{OH}) 3 \mathrm{O}-$ \\
\hline 24 & 59 & 87 & 49 & 85 & 49 & 87 & 56 & 83 & esp29 & $\leftrightarrow$ & esp30 & ESP24 & $\mathrm{H} 2 \mathrm{O}$ \\
\hline 25 & 0 & 13 & 0 & 18 & 0 & 20 & 0 & 21 & esp 30 & $\leftrightarrow$ & esp31+esp24 & ESP25 & $\mathrm{OH}-$ \\
\hline 26 & 49 & 4 & 50 & 5 & 52 & 1 & 58 & 16 & esp31 & $\leftrightarrow$ & esp32 & ESP26 & $\mathrm{H} 3 \mathrm{O}+$ \\
\hline 27 & 0 & 84 & 0 & 53 & 0 & 73 & 0 & 83 & esp32 & $\leftrightarrow$ & esp 33 & ESP27 & $\mathrm{H}+$ \\
\hline 28 & 0 & 0 & 0 & 0 & 0 & 0 & 0 & 0 & esp15 + esp22 & $\leftrightarrow$ & esp34+esp24 & ESP28 & {$\left[\mathrm{Si}(\mathrm{OH}) 3 \mathrm{O}_{, \prime,}, \mathrm{Si} 4 \mathrm{O} 13 \mathrm{H} 10\right]-$} \\
\hline 29 & 46 & 31 & 52 & 42 & 48 & 43 & 54 & 39 & esp 34 & $\leftrightarrow$ & esp35 & ESP29 & {$[[\mathrm{Si}(\mathrm{OH}) 3-\mathrm{O}-\mathrm{Si} 4 \mathrm{O} 13 \mathrm{H} 10]-]-$} \\
\hline 30 & 25 & 77 & 13 & 63 & 18 & 46 & 35 & 71 & esp35 & $\leftrightarrow$ & esp 36 & ESP30 & Si5O16H11-...H2O \\
\hline 31 & 0 & 21 & 0 & 14 & 0 & 30 & 0 & 10 & esp $30+$ esp 22 & $\leftrightarrow$ & esp37 + esp24 & ESP31 & Si5015H9-(сус) \\
\hline 32 & 47 & 33 & 45 & 23 & 48 & 24 & 43 & 28 & esp 37 & $\leftrightarrow$ & esp38 & ESP32 & [Si5015H9-]-(cyc) \\
\hline 33 & 59 & 87 & 49 & 85 & 49 & 87 & 56 & 83 & esp38 & $\leftrightarrow$ & esp39 & ESP33 & 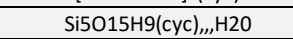 \\
\hline 34 & 0 & 13 & 0 & 18 & 0 & 20 & 0 & 21 & esp39 & $\leftrightarrow$ & esp40+esp24 & ESP34 & {$\left[\mathrm{Si}(\mathrm{OH}) 3 \mathrm{O}_{, \ldots,, \mathrm{S}} \mathrm{S} 4 \mathrm{O} 13 \mathrm{H} 1 \mathrm{O}\right]-(+)$} \\
\hline 35 & 19 & 19 & 40 & 6 & 52 & 16 & 37 & 9 & esp 40 & $\leftrightarrow$ & esp41 & ESP35 & {$[\mathrm{Si}(\mathrm{OH}) 3 \mathrm{O}-\mathrm{Si} 4 \mathrm{O} 13 \mathrm{H} 10]-(+)$} \\
\hline 36 & 33 & 57 & 26 & 63 & 29 & 64 & 22 & 60 & esp 41 & $\leftrightarrow$ & esp 42 & ESP36 & Si5016H11-...H2O (-) \\
\hline 37 & 0 & 21 & 0 & 14 & 0 & 30 & 0 & 10 & esp39+ esp22 & $\leftrightarrow$ & esp45+ esp24 & ESP37 & {$\left[\mathrm{Si}(\mathrm{OH}) 3 \mathrm{O}_{,, \ldots,}, \mathrm{Si} 5 \mathrm{O} 16 \mathrm{H} 12\right]-$} \\
\hline 38 & 47 & 33 & 45 & 23 & 48 & 24 & 43 & 28 & esp 45 & $\leftrightarrow$ & esp 46 & ESP38 & {$[\mathrm{Si}(\mathrm{OH}) 3 \mathrm{O}-\mathrm{Si} 5 \mathrm{O} 16 \mathrm{H} 12]^{-}$} \\
\hline 39 & 59 & 87 & 49 & 85 & 49 & 87 & 56 & 83 & esp 46 & $\leftrightarrow$ & esp 47 & ESP39 & Si6O19H13-...H2O \\
\hline 40 & 0 & 21 & 0 & 14 & 0 & 30 & 0 & 10 & esp $47+$ esp 22 & $\leftrightarrow$ & esp48 +esp24 & $\overline{E S P 40}$ & Si6019H13-(сус) \\
\hline 41 & 47 & 33 & 45 & 23 & 48 & 24 & 43 & 28 & esp 48 & $\leftrightarrow$ & esp 49 & ESP41 & [Si6019H13-](сус) \\
\hline 42 & 59 & 87 & 49 & 85 & 49 & 87 & 56 & 83 & esp 49 & $\leftrightarrow$ & esp50 & ESP42 & Si6O18H11(сус)-,,, $\mathrm{H}_{2} 20$ \\
\hline 43 & 0 & 30 & 0 & 50 & 0 & 66 & 0 & 44 & esp $47+$ esp 22 & $\leftrightarrow$ & esp51 +esp24 & $\overline{E S P 43}$ & Si2O7H6...Si2O7H5- \\
\hline 44 & 52 & 47 & 70 & 37 & 72 & 36 & 68 & 41 & esp51 & $\leftrightarrow$ & esp52 & ESP44 & [Si2O6H5-O-Si2O7H6]- \\
\hline 45 & 48 & 82 & 44 & 85 & 40 & 80 & 44 & 84 & esp52 & $\leftrightarrow$ & esp53 & ESP45 & Heptamer reactif complex \\
\hline
\end{tabular}




\begin{tabular}{|c|c|c|c|c|c|c|c|c|c|c|c|c|c|}
\hline 46 & 0 & 30 & 0 & 50 & 0 & 66 & 0 & 44 & \multirow{3}{*}{$\begin{array}{c}\text { esp } 47+\text { esp22 } \\
\text { esp54 } \\
\text { esp55 }\end{array}$} & $\leftrightarrow$ & \multirow{3}{*}{$\begin{array}{c}\text { esp54 + esp24 } \\
\text { esp55 } \\
\text { esp56 }\end{array}$} & \multirow{3}{*}{$\begin{array}{l}\text { ESP46 } \\
\text { ESP47 } \\
\text { ESP48 }\end{array}$} & Heptamer intermediate \\
\hline 47 & 52 & 47 & 70 & 37 & 72 & 36 & 68 & 41 & & $\leftrightarrow$ & & & Heptamer...H2O \\
\hline 48 & 48 & 82 & 44 & 85 & 40 & 80 & 44 & 84 & & $\leftrightarrow$ & & & Octamer reactif complex \\
\hline 49 & 0 & 30 & 0 & 50 & 0 & 66 & 0 & 44 & \multirow{3}{*}{$\begin{array}{c}\text { esp } 47+\text { esp22 } \\
\text { esp57 } \\
\text { esp58 }\end{array}$} & $\leftrightarrow$ & \multirow{3}{*}{$\begin{array}{c}\text { esp57 + esp24 } \\
\text { esp58 } \\
\text { esp59 }\end{array}$} & \multirow{3}{*}{$\begin{array}{l}\text { ESP49 } \\
\text { ESP50 } \\
\text { ESP51 }\end{array}$} & Octamer intermediate \\
\hline 50 & 52 & 47 & 70 & 37 & 72 & 36 & 68 & 41 & & $\leftrightarrow$ & & & Octamer Hydrated_product \\
\hline 51 & 48 & 82 & 44 & 85 & 40 & 80 & 44 & 84 & & $\leftrightarrow$ & & & Heptamer -2-beanched-reactif-complex \\
\hline 52 & 0 & 21 & 0 & 14 & 0 & 30 & 0 & 10 & \multirow{3}{*}{$\begin{array}{c}\text { esp15 + esp22 } \\
\text { esp60 } \\
\text { esp61 } \\
\end{array}$} & $\leftrightarrow$ & \multirow{3}{*}{$\begin{array}{c}\text { esp60 + esp24 } \\
\text { esp61 } \\
\text { esp62 }\end{array}$} & \multirow{3}{*}{$\begin{array}{l}\text { ESP52 } \\
\text { ESP53 } \\
\text { ESP54 } \\
\end{array}$} & Hepta -2-branched_Intermediate \\
\hline 53 & 47 & 33 & 45 & 23 & 48 & 24 & 43 & 28 & & $\leftrightarrow$ & & & Hepta -2-branched...H2O \\
\hline 54 & 59 & 87 & 49 & 85 & 49 & 87 & 56 & 83 & & $\leftrightarrow$ & & & Hepta -3-branched_reactif_complex \\
\hline 55 & 0 & 30 & 0 & 50 & 0 & 66 & 0 & 44 & \multirow{3}{*}{$\begin{array}{c}\text { esp } 15+\text { esp3 } \\
\text { esp63 } \\
\text { esp64 }\end{array}$} & $\leftrightarrow$ & \multirow{3}{*}{$\begin{array}{c}\text { esp63 + esp24 } \\
\text { esp64 } \\
\text { esp62 }\end{array}$} & \multirow{3}{*}{$\begin{array}{l}\text { ESP55 } \\
\text { ESP56 } \\
\text { ESP57 }\end{array}$} & Hepta -3-branched_Intermediate \\
\hline 56 & 52 & 47 & 70 & 37 & 72 & 36 & 68 & 41 & & $\leftrightarrow$ & & & Hepta -3-branched...H2O \\
\hline 57 & 48 & 82 & 44 & 85 & 40 & 80 & 44 & 84 & & $\leftrightarrow$ & & & Isoheptamer_reactif_complex \\
\hline 58 & 0 & 30 & 0 & 50 & 0 & 66 & 0 & 44 & \multirow{3}{*}{$\begin{array}{c}\text { esp } 30+\text { esp22 } \\
\text { esp242 } \\
\text { esp65 }\end{array}$} & $\leftrightarrow$ & \multirow{3}{*}{$\begin{array}{c}\text { esp242 + esp24 } \\
\text { esp65 } \\
\text { esp66 }\end{array}$} & \multirow{3}{*}{$\begin{array}{l}\text { ESP58 } \\
\text { ESP59 } \\
\text { ESP60 } \\
\end{array}$} & Isoheptamer_Intermediate \\
\hline 59 & 52 & 47 & 70 & 37 & 72 & 36 & 68 & 41 & & $\leftrightarrow$ & & & Isoheptamer... $\mathrm{H} 2 \mathrm{O}$ \\
\hline 60 & 48 & 82 & 44 & 85 & 40 & 80 & 44 & 84 & & $\leftrightarrow$ & & & Tetramer_2_branched_reactif_complex \\
\hline 61 & 0 & 21 & 0 & 14 & 0 & 30 & 0 & 10 & \multirow{3}{*}{$\begin{array}{c}\text { esp62 + esp22 } \\
\text { esp67 } \\
\text { esp68 }\end{array}$} & $\leftrightarrow$ & \multirow{3}{*}{$\begin{array}{c}\text { esp67 + esp24 } \\
\text { esp68 } \\
\text { esp66 }\end{array}$} & \multirow{3}{*}{$\begin{array}{l}\text { ESP61 } \\
\text { ESP62 } \\
\text { ESP63 }\end{array}$} & Tetramer2branched_Intermediate \\
\hline 62 & 47 & 33 & 45 & 23 & 48 & 24 & 43 & 28 & & $\leftrightarrow$ & & & Tetramer2branched_Product \\
\hline 63 & 59 & 87 & 49 & 85 & 49 & 87 & 56 & 83 & & $\leftrightarrow$ & & & Tetramer2branched_reactif_complex \\
\hline 64 & 0 & 30 & 0 & 50 & 0 & 66 & 0 & 44 & & $\leftrightarrow$ & esp69 + esp24 & ESP64 & Isopentamer_reactif_complex \\
\hline 65 & 52 & 47 & 70 & 37 & 72 & 36 & 68 & 41 & esp69 & $\leftrightarrow$ & esp70 & ESP65 & Isopentamer_Intermediate \\
\hline 66 & 48 & 82 & 44 & 85 & 40 & 80 & 44 & 84 & & $\leftrightarrow$ & esp71 & ESP66 & Isopentamer...H2O \\
\hline 67 & 0 & 21 & 0 & 14 & 0 & 30 & 0 & 10 & esp62 + esp22 & $\leftrightarrow$ & esp72 + esp24 & ESP67 & Isopentamer_reactif_complex \\
\hline 68 & 47 & 33 & 45 & 23 & 48 & 24 & 43 & 28 & esp72 & $\leftrightarrow$ & esp73 & ESP68 & Isopentamer_Intermediate \\
\hline 69 & 59 & 87 & 49 & 85 & 49 & 87 & 56 & 83 & esp 73 & $\leftrightarrow$ & esp71 & ESP69 & Pentamer2branched_reactif_complex \\
\hline 70 & 0 & 30 & 0 & 50 & 0 & 66 & 0 & 44 & esp62 + esp22 & $\leftrightarrow$ & esp74 + esp24 & ESP70 & Pentamer2branched_Intermediate \\
\hline 71 & 52 & 47 & 70 & 37 & 72 & 36 & 68 & 41 & esp74 & $\leftrightarrow$ & esp75 & ESP71 & Pentamer2branched_product \\
\hline 72 & 48 & 82 & 44 & 85 & 40 & 80 & 44 & 84 & esp75 & $\leftrightarrow$ & esp76 & ESP72 & Pentamer2branched_reactif_complex_v2 \\
\hline 73 & 0 & 0 & 0 & 0 & 0 & 0 & 0 & 0 & esp62 + esp22 & $\leftrightarrow$ & esp77 + esp24 & ESP73 & Pentamer2branched_Intermediate_V2 \\
\hline 74 & 46 & 31 & 52 & 42 & 48 & 43 & 54 & 39 & esp77 & $\leftrightarrow$ & esp78 & ESP74 & 2,3Tetramerbranched_reactif_complex \\
\hline 75 & 25 & 77 & 13 & 63 & 18 & 46 & 35 & 71 & esp 78 & $\leftrightarrow$ & esp79 & ESP75 & 2,3Tetramerbranched_Intermediate \\
\hline 76 & 0 & 21 & 0 & 14 & 0 & 30 & 0 & 10 & esp36 + esp22 & $\leftrightarrow$ & esp80 + esp24 & ESP76 & 2,3Tetramerbranched...H2O \\
\hline 77 & 47 & 33 & 45 & 23 & 48 & 24 & 43 & 28 & esp80 & $\leftrightarrow$ & esp81 & ESP77 & 2,2Tetramerbranched_reactif_complex \\
\hline 78 & 59 & 87 & 49 & 85 & 49 & 87 & 56 & 83 & esp 81 & $\leftrightarrow$ & esp79 & ESP78 & 2,2Tetramerbranched_Intermediate \\
\hline 79 & 0 & 21 & 0 & 14 & 0 & 30 & 0 & 10 & esp76 + esp22 & $\leftrightarrow$ & esp82 + esp24 & ESP79 & 2,2Tetramerbranched_product \\
\hline 80 & 47 & 33 & 45 & 23 & 48 & 24 & 43 & 28 & esp82 & $\leftrightarrow$ & esp83 & ESP80 & 2,2Tetramerbranched_reactif_complex_V2 \\
\hline 81 & 59 & 87 & 49 & 85 & 49 & 87 & 56 & 83 & esp 83 & $\leftrightarrow$ & esp84 & ESP81 & 2,2Tetramerbranched_Intermediate_V2 \\
\hline 82 & 0 & 30 & 0 & 50 & 0 & 66 & 0 & 44 & esp71 + esp22 & $\leftrightarrow$ & esp85 + esp24 & ESP82 & 2,3pentamerbranched_reactif_complex \\
\hline 83 & 52 & 47 & 70 & 37 & 72 & 36 & 68 & 41 & esp85 & $\leftrightarrow$ & esp86 & ESP83 & 2,3pentamerbranched_Intermediate \\
\hline 84 & 48 & 82 & 44 & 85 & 40 & 80 & 44 & 84 & esp 86 & $\leftrightarrow$ & esp 84 & ESP84 & 2,3pentamerbranched...H2O \\
\hline 85 & 0 & 30 & 0 & 50 & 0 & 66 & 0 & 44 & esp66 + esp22 & $\leftrightarrow$ & esp87 + esp24 & ESP85 & 2,3pentamerbranched_reactif_complex- $\mathrm{V} 2$ \\
\hline 86 & 52 & 47 & 70 & 37 & 72 & 36 & 68 & 41 & esp87 & $\leftrightarrow$ & esp88 & ESP86 & 2,3pentamerbranched_Intermediate_V2 \\
\hline 87 & 48 & 82 & 44 & 85 & 40 & 80 & 44 & 84 & esp 88 & $\leftrightarrow$ & esp84 & ESP87 & 2,3pentamerbranched_reactif_complex-V33 \\
\hline 88 & 0 & 21 & 0 & 14 & 0 & 30 & 0 & 10 & esp66 + esp22 & $\leftrightarrow$ & esp89 + esp24 & ESP88 & 2,3pentamerbranched_Intermediate_V3 \\
\hline 89 & 47 & 33 & 45 & 23 & 48 & 24 & 43 & 28 & esp89 & $\leftrightarrow$ & esp90 & ESP89 & 3dimerpentamerbranched_reactif_complex \\
\hline 90 & 59 & 87 & 49 & 85 & 49 & 87 & 56 & 83 & esp90 & $\leftrightarrow$ & esp91 & ESP90 & 3dimerpentamerbranched_Intermediate \\
\hline 91 & 0 & 30 & 0 & 50 & 0 & 66 & 0 & 44 & esp39+esp22 & $\leftrightarrow$ & esp92 + esp24 & ESP91 & 3dimerpentamerbranched... $\mathrm{H} 2 \mathrm{O}$ \\
\hline 92 & 52 & 47 & 70 & 37 & 72 & 36 & 68 & 41 & esp92 & $\leftrightarrow$ & esp93 & ESP92 & 3hexamerbranched_reactif_complex \\
\hline 93 & 48 & 82 & 44 & 85 & 40 & 80 & 44 & 84 & esp93 & $\leftrightarrow$ & esp94 & ESP93 & 3hexamerbranched_Intermediate \\
\hline
\end{tabular}




\begin{tabular}{|c|c|c|c|c|c|c|c|c|c|c|c|c|c|}
\hline 94 & 0 & 21 & 0 & 14 & 0 & 30 & 0 & 10 & \multirow{3}{*}{$\begin{array}{c}\text { esp71 + esp22 } \\
\text { esp95 } \\
\text { esp96 }\end{array}$} & $\leftrightarrow$ & \multirow{3}{*}{$\begin{array}{c}\text { esp95 + esp24 } \\
\text { esp96 } \\
\text { esp94 }\end{array}$} & \multirow{3}{*}{$\begin{array}{l}\text { ESP94 } \\
\text { ESP95 } \\
\text { ESP96 } \\
\end{array}$} & 3hexamerbranched... $\mathrm{H} 2 \mathrm{O}$ \\
\hline 95 & 47 & 33 & 45 & 23 & 48 & 24 & 43 & 28 & & $\leftrightarrow$ & & & 3hexamerbranched_reactif_complex_V2 \\
\hline 96 & 59 & 87 & 49 & 85 & 49 & 87 & 56 & 83 & & $\leftrightarrow$ & & & 3hexamerbranched_Intermediate_V2 \\
\hline 97 & 0 & 21 & 0 & 14 & 0 & 30 & 0 & 10 & \multirow{3}{*}{$\begin{array}{c}\text { esp66 + esp22 } \\
\text { esp97 } \\
\text { esp98 }\end{array}$} & $\leftrightarrow$ & \multirow{3}{*}{$\begin{array}{c}\text { esp97 + esp24 } \\
\text { esp98 } \\
\text { esp94 } \\
\end{array}$} & \multirow{3}{*}{$\begin{array}{l}\text { ESP97 } \\
\text { ESP98 } \\
\text { ESP99 }\end{array}$} & 3hexamerbranched_reactif_complex_V3 \\
\hline 98 & 47 & 33 & 45 & 23 & 48 & 24 & 43 & 28 & & $\leftrightarrow$ & & & 3hexamerbranched_Intermediate_V3 \\
\hline 99 & 59 & 87 & 49 & 85 & 49 & 87 & 56 & 83 & & $\leftrightarrow$ & & & 2hexamerbranched_reactif_complex \\
\hline 100 & 0 & 30 & 0 & 50 & 0 & 66 & 0 & 44 & \multirow{3}{*}{$\begin{array}{c}\text { esp39 + esp22 } \\
\text { esp99 } \\
\text { esp100 }\end{array}$} & $\leftrightarrow$ & \multirow{3}{*}{$\begin{array}{c}\text { esp99 + esp24 } \\
\text { esp100 } \\
\text { esp101 }\end{array}$} & \multirow{3}{*}{$\begin{array}{l}\text { ESP100 } \\
\text { ESP101 } \\
\text { ESP102 } \\
\end{array}$} & 2hexamerbranched_Intermediate \\
\hline 101 & 52 & 47 & 70 & 37 & 72 & 36 & 68 & 41 & & $\leftrightarrow$ & & & 2hexamerbranched... $\mathrm{H} 2 \mathrm{O}$ \\
\hline 102 & 48 & 82 & 44 & 85 & 40 & 80 & 44 & 84 & & $\leftrightarrow$ & & & 2hexamerbranched_reactif_complex_V2 \\
\hline 103 & 0 & 21 & 0 & 14 & 0 & 30 & 0 & 10 & \multirow{3}{*}{$\begin{array}{c}\text { esp71 + esp22 } \\
\text { esp102 } \\
\text { esp103 }\end{array}$} & $\leftrightarrow$ & \multirow{3}{*}{$\begin{array}{c}\text { esp102 + esp24 } \\
\text { esp103 } \\
\text { esp101 }\end{array}$} & \multirow{3}{*}{$\begin{array}{l}\text { ESP103 } \\
\text { ESP104 } \\
\text { ESP105 }\end{array}$} & 2hexamerbranched_Intermediate_V2 \\
\hline 104 & 47 & 33 & 45 & 23 & 48 & 24 & 43 & 28 & & $\leftrightarrow$ & & & 2,3,3Tetramer_reactif_complex \\
\hline 105 & 59 & 87 & 49 & 85 & 49 & 87 & 56 & 83 & & $\leftrightarrow$ & & & 2,3,3Tetramer_Intermediate \\
\hline 106 & 0 & 30 & 0 & 50 & 0 & 66 & 0 & 44 & \multirow{3}{*}{$\begin{array}{c}\text { esp79 + esp22 } \\
\text { esp } 104 \\
\text { esp105 }\end{array}$} & $\leftrightarrow$ & \multirow{3}{*}{$\begin{array}{c}\text { esp104 + esp24 } \\
\text { esp105 } \\
\text { esp106 } \\
\end{array}$} & \multirow{3}{*}{$\begin{array}{l}\text { ESP106 } \\
\text { ESP107 } \\
\text { ESP108 }\end{array}$} & 2,3,3Tetramer...H2O \\
\hline 107 & 52 & 47 & 70 & 37 & 72 & 36 & 68 & 41 & & $\leftrightarrow$ & & & 2,3,3Tetramer_reactif_complex_V2 \\
\hline 108 & 48 & 82 & 44 & 85 & 40 & 80 & 44 & 84 & & $\leftrightarrow$ & & & 2,3,3Tetramer_Intermediate_V2 \\
\hline 109 & 0 & 0 & 0 & 0 & 0 & 0 & 0 & 0 & \multirow{3}{*}{$\begin{array}{c}\text { esp } 76+\text { e esp22 } \\
\text { esp } 107 \\
\text { esp108 } \\
\end{array}$} & $\leftrightarrow$ & \multirow{3}{*}{$\begin{array}{c}\text { esp107 + esp24 } \\
\text { esp108 } \\
\text { esp106 } \\
\end{array}$} & \multirow{3}{*}{$\begin{array}{l}\text { ESP109 } \\
\text { ESP110 } \\
\text { ESP111 }\end{array}$} & 4-ring2-(trimer)branched_reactif_complex \\
\hline 110 & 46 & 31 & 52 & 42 & 48 & 43 & 54 & 39 & & $\leftrightarrow$ & & & 4-ring2-(trimer)branched_Intermediate \\
\hline 111 & 25 & 77 & 13 & 63 & 18 & 46 & 35 & 71 & & $\leftrightarrow$ & & & 4-ring2-(trimer)branched... $\mathrm{H} 2 \mathrm{O}$ \\
\hline$\overline{112}$ & 0 & 13 & 0 & 18 & 0 & 20 & 0 & 21 & esp84 & $\leftrightarrow$ & esp109 + esp24 & ESP112 & 4-ring2-(trimer)branched_reactif_complex_V2 \\
\hline 113 & 28 & 16 & 28 & 15 & 29 & 17 & 25 & 18 & esp109 & $\leftrightarrow$ & esp110 & ESP113 & 4-ring2-(trimer)branched_Intermediate_V2 \\
\hline 114 & 48 & 75 & 44 & 79 & 50 & 83 & 57 & 79 & esp110 & $\leftrightarrow$ & esp111 & ESP114 & 3,3isopentamerbranched_reactif_complex \\
\hline 115 & 0 & 13 & 0 & 18 & 0 & 20 & 0 & 21 & esp102 & $\leftrightarrow$ & esp112+ esp24 & ESP115 & 3,3isopentamerbranched_Intermediate \\
\hline 116 & 28 & 16 & 28 & 15 & 29 & 17 & 25 & 18 & esp112 & $\leftrightarrow$ & esp113 & ESP116 & 3,3isopentamerbranched... $\mathrm{H} 2 \mathrm{O}$ \\
\hline 117 & 48 & 75 & 44 & 79 & 50 & 83 & 57 & 79 & esp113 & $\leftrightarrow$ & esp111 & ESP117 & 3,3isopentamerbranched_reactif_complex_V2 \\
\hline 118 & 0 & 0 & 0 & 0 & 0 & 0 & 0 & 0 & esp $66+$ esp 22 & $\leftrightarrow$ & esp114 + esp24 & ESP118 & 3,3isopentamerbranched_Intermediate_V2 \\
\hline 119 & 46 & 31 & 52 & 42 & 48 & 43 & 54 & 39 & esp114 & $\leftrightarrow$ & esp115 & ESP119 & 2,2pentamerbranched_reactif_complex \\
\hline 120 & 25 & 77 & 13 & 63 & 18 & 46 & 35 & 71 & esp115 & $\leftrightarrow$ & esp116 & ESP120 & 2,2pentamerbranched_Intermediate \\
\hline$\overline{121}$ & 0 & 21 & 0 & 14 & 0 & 30 & 0 & 10 & esp79+esp22 & $\leftrightarrow$ & esp117 + esp24 & ESP121 & 2,2pentamerbranched... $\mathrm{H} 2 \mathrm{O}$ \\
\hline 122 & 47 & 33 & 45 & 23 & 48 & 24 & 43 & 28 & esp117 & $\leftrightarrow$ & esp118 & ESP122 & 2,2pentamerbranched_reactif_complex_V2 \\
\hline 123 & 59 & 87 & 49 & 85 & 49 & 87 & 56 & 83 & esp118 & $\leftrightarrow$ & esp116 & ESP123 & 2,2pentamerbranched_Intermediate_V2 \\
\hline$\overline{124}$ & 0 & 0 & 0 & 0 & 0 & 0 & 0 & 0 & esp71+esp22 & $\leftrightarrow$ & esp119 + esp24 & ESP124 & 2,4pentamerbranched_reactif_complex \\
\hline 125 & 46 & 31 & 52 & 42 & 48 & 43 & 54 & 39 & esp119 & $\leftrightarrow$ & esp120 & ESP125 & 2,4pentamerbranched_Intermediate \\
\hline 126 & 25 & 77 & 13 & 63 & 18 & 46 & 35 & 71 & esp120 & $\leftrightarrow$ & esp121 & ESP126 & 2,4pentamerbranched... $\mathrm{H} 2 \mathrm{O}$ \\
\hline 127 & 0 & 21 & 0 & 14 & 0 & 30 & 0 & 10 & esp79+ esp22 & $\leftrightarrow$ & esp122 + esp24 & ESP127 & 3heptamerbranched_reactif_complex \\
\hline 128 & 47 & 33 & 45 & 23 & 48 & 24 & 43 & 28 & esp122 & $\leftrightarrow$ & esp123 & ESP128 & 3heptamerbranched_Intermediate \\
\hline 129 & 59 & 87 & 49 & 85 & 49 & 87 & 56 & 83 & esp123 & $\leftrightarrow$ & esp121 & ESP129 & 3heptamerbranched_reactif_complex_V2 \\
\hline 130 & 0 & 30 & 0 & 50 & 0 & 66 & 0 & 44 & esp71+esp22 & $\leftrightarrow$ & esp124 + esp24 & ESP130 & 3heptamerbranched_Intermediate_V2 \\
\hline 131 & 52 & 47 & 70 & 37 & 72 & 36 & 68 & 41 & esp124 & $\leftrightarrow$ & esp125 & ESP131 & Isoheptamerbranched_reactif_complex \\
\hline 132 & 48 & 82 & 44 & 85 & 40 & 80 & 44 & 84 & esp125 & $\leftrightarrow$ & esp126 & ESP132 & Isoheptamerbranched_Intermediate \\
\hline 133 & 0 & 21 & 0 & 14 & 0 & 30 & 0 & 10 & esp94 + esp22 & $\leftrightarrow$ & esp127 + esp24 & ESP133 & Isoheptamerbranched... $\mathrm{H} 2 \mathrm{O}$ \\
\hline 134 & 47 & 33 & 45 & 23 & 48 & 24 & 43 & 28 & esp127 & $\leftrightarrow$ & esp128 & ESP134 & 2,4hexamerbranched_reactif_complex \\
\hline 135 & 59 & 87 & 49 & 85 & 49 & 87 & 56 & 83 & esp128 & $\leftrightarrow$ & esp56 & ESP135 & 2,4hexamerbranched_Intermediate \\
\hline 136 & 0 & 21 & 0 & 14 & 0 & 30 & 0 & 10 & esp101 + esp22 & $\leftrightarrow$ & esp129 + esp24 & ESP136 & 2,4hexamerbranched... H2O \\
\hline 137 & 47 & 33 & 45 & 23 & 48 & 24 & 43 & 28 & esp129 & $\leftrightarrow$ & esp130 & ESP137 & 2,4hexamerbranched_reactif_complex_V2 \\
\hline 138 & 59 & 87 & 49 & 85 & 49 & 87 & 56 & 83 & esp130 & $\leftrightarrow$ & esp56 & ESP138 & 2,4hexamerbranched_Intermediate_V2 \\
\hline 139 & 0 & 21 & 0 & 14 & 0 & 30 & 0 & 10 & esp94 + esp22 & $\leftrightarrow$ & esp131+ esp24 & ESP139 & 3,4hexamerbranched_reactif_complex \\
\hline 140 & 47 & 33 & 45 & 23 & 48 & 24 & 43 & 28 & esp131 & $\leftrightarrow$ & esp132 & ESP140 & 3,4hexamerbranched_Intermediate \\
\hline 141 & 59 & 87 & 49 & 85 & 49 & 87 & 56 & 83 & esp132 & $\leftrightarrow$ & esp133 & ESP141 & 3,4hexamerbranched... $\mathrm{H} 2 \mathrm{O}$ \\
\hline
\end{tabular}




\begin{tabular}{|c|c|c|c|c|c|c|c|c|c|c|c|c|c|}
\hline 142 & 0 & 30 & 0 & 50 & 0 & 66 & 0 & 44 & \multirow{3}{*}{$\begin{array}{c}\text { esp47 + esp22 } \\
\text { esp239 } \\
\text { esp240 }\end{array}$} & $\leftrightarrow$ & \multirow{3}{*}{$\begin{array}{l}\text { esp239 + esp24 } \\
\text { esp240 } \\
\text { esp133 }\end{array}$} & \multirow{3}{*}{$\begin{array}{l}\text { ESP142 } \\
\text { ESP143 } \\
\text { ESP144 }\end{array}$} & 4,4hexamerbranched_reactif_complex \\
\hline 143 & 52 & 47 & 70 & 37 & 72 & 36 & 68 & 41 & & $\leftrightarrow$ & & & 4,4hexamerbranched_Intermediate \\
\hline 144 & 48 & 82 & 44 & 85 & 40 & 80 & 44 & 84 & & $\leftrightarrow$ & & & 4,4hexamerbranched... $\mathrm{H} 2 \mathrm{O}$ \\
\hline 145 & 0 & 30 & 0 & 50 & 0 & 66 & 0 & 44 & \multirow{3}{*}{$\begin{array}{c}\text { esp101+ esp22 } \\
\text { esp134 } \\
\text { esp135 } \\
\end{array}$} & $\leftrightarrow$ & \multirow{3}{*}{$\begin{array}{c}\text { esp134 + esp24 } \\
\text { esp135 } \\
\text { esp136 } \\
\end{array}$} & \multirow{3}{*}{$\begin{array}{l}\text { ESP145 } \\
\text { ESP146 } \\
\text { ESP147 }\end{array}$} & 4,5hexamerbranched_reactif_complex \\
\hline 146 & 52 & 47 & 70 & 37 & 72 & 36 & 68 & 41 & & $\leftrightarrow$ & & & 4,5hexamerbranched_Intermediate \\
\hline 147 & 48 & 82 & 44 & 85 & 40 & 80 & 44 & 84 & & $\leftrightarrow$ & & & 4,5hexamerbranched... $\mathrm{H} 2 \mathrm{O}$ \\
\hline 148 & 0 & 30 & 0 & 50 & 0 & 66 & 0 & 44 & \multirow{3}{*}{$\begin{array}{c}\text { esp94 + esp22 } \\
\text { esp137 } \\
\text { esp138 } \\
\end{array}$} & $\leftrightarrow$ & \multirow{3}{*}{$\begin{array}{c}\text { esp137 + esp24 } \\
\text { esp138 } \\
\text { esp136 } \\
\end{array}$} & \multirow{3}{*}{$\begin{array}{l}\text { ESP148 } \\
\text { ESP149 } \\
\text { ESP150 } \\
\end{array}$} & 2heptamerbranched_reactif_complex \\
\hline 149 & 52 & 47 & 70 & 37 & 72 & 36 & 68 & 41 & & $\leftrightarrow$ & & & 2heptamerbranched_Intermediate \\
\hline 150 & 48 & 82 & 44 & 85 & 40 & 80 & 44 & 84 & & $\leftrightarrow$ & & & 2heptamerbranched... $\mathrm{H} 2 \mathrm{O}$ \\
\hline 151 & 0 & 30 & 0 & 50 & 0 & 66 & 0 & 44 & \multirow{3}{*}{$\begin{array}{c}\text { esp94 + esp22 } \\
\text { esp139 } \\
\text { esp140 } \\
\end{array}$} & $\leftrightarrow$ & \multirow{3}{*}{$\begin{array}{c}\text { esp139+ esp24 } \\
\text { esp140 } \\
\text { esp141 } \\
\end{array}$} & \multirow{3}{*}{$\begin{array}{l}\text { ESP151 } \\
\text { ESP152 } \\
\text { ESP153 } \\
\end{array}$} & 2,5hexamerbranched_reactif_complex \\
\hline 152 & 52 & 47 & 70 & 37 & 72 & 36 & 68 & 41 & & $\leftrightarrow$ & & & 2,5hexamerbranched_Intermediate \\
\hline 153 & 48 & 82 & 44 & 85 & 40 & 80 & 44 & 84 & & $\leftrightarrow$ & & & 2,5hexamerbranched...H2O \\
\hline 154 & 0 & 0 & 0 & 0 & 0 & 0 & 0 & 0 & \multirow{3}{*}{$\begin{array}{c}\text { esp94 + esp22 } \\
\text { esp142 } \\
\end{array}$} & $\leftrightarrow$ & \multirow{3}{*}{$\begin{array}{c}\text { esp142 + esp24 } \\
\text { esp143 } \\
\text { esp144 }\end{array}$} & \multirow{3}{*}{$\begin{array}{l}\text { ESP154 } \\
\text { ESP155 } \\
\text { ESP156 }\end{array}$} & 4,5hexamerbranched_reactif_complex \\
\hline 155 & 46 & 31 & 52 & 42 & 48 & 43 & 54 & 39 & & $\leftrightarrow$ & & & 4,5hexamerbranched_Intermediate \\
\hline 156 & 25 & 77 & 13 & 63 & 18 & 46 & 35 & 71 & & $\leftrightarrow$ & & & 2heptamer-branched_reactif_complex \\
\hline$\overline{157}$ & 0 & 30 & 0 & 50 & 0 & 66 & 0 & 44 & \multirow{3}{*}{$\begin{array}{l}\text { esp94 + esp22 } \\
\text { esp145 } \\
\text { esp146 }\end{array}$} & $\leftrightarrow$ & \multirow{3}{*}{$\begin{array}{c}\text { esp145 + esp24 } \\
\text { esp146 } \\
\text { esp147 } \\
\end{array}$} & \multirow{3}{*}{$\begin{array}{l}\text { ESP157 } \\
\text { ESP158 } \\
\text { ESP159 }\end{array}$} & 2heptamer-branched_Intermediate \\
\hline 158 & 52 & 47 & 70 & 37 & 72 & 36 & 68 & 41 & & $\leftrightarrow$ & & & 4-ring2,3branched(3-dimer)_reactif_complex \\
\hline 159 & 48 & 82 & 44 & 85 & 40 & 80 & 44 & 84 & & $\leftrightarrow$ & & & 4-ring2,3branched(3-dimer)_Intermediate \\
\hline 160 & 0 & 21 & 0 & 14 & 0 & 30 & 0 & 10 & & $\leftrightarrow$ & esp148+esp24 & ESP160 & 4-ring2,3branched(3-dimer)...H2O \\
\hline 161 & 47 & 33 & 45 & 23 & 48 & 24 & 43 & 28 & esp148 & $\leftrightarrow$ & esp149 & ESP161 & 2,3,4pentamerbranched_reactif_complex \\
\hline 162 & 59 & 87 & 49 & 85 & 49 & 87 & 56 & 83 & esp149 & $\leftrightarrow$ & esp150 & ESP162 & 2,3,4pentamerbranched_Intermediate \\
\hline 163 & 0 & 30 & 0 & 50 & 0 & 66 & 0 & 44 & esp101+esp22 & $\leftrightarrow$ & esp151+esp24 & ESP163 & 2,3,4pentamerbranched...H2O \\
\hline 164 & 52 & 47 & 70 & 37 & 72 & 36 & 68 & 41 & esp151 & $\leftrightarrow$ & esp152 & ESP164 & 2,3hexamerbranched_reactif_complex \\
\hline 165 & 48 & 82 & 44 & 85 & 40 & 80 & 44 & 84 & esp152 & $\leftrightarrow$ & esp153 & ESP165 & 2,3hexamerbranched_Intermediate \\
\hline 166 & 0 & 30 & 0 & 50 & 0 & 66 & 0 & 44 & esp101+ esp22 & $\leftrightarrow$ & esp154 + esp24 & ESP166 & 2,3hexamerbranched... $\mathrm{H} 2 \mathrm{O}$ \\
\hline 167 & 52 & 47 & 70 & 37 & 72 & 36 & 68 & 41 & esp154 & $\leftrightarrow$ & esp155 & ESP167 & 3,4hexamerbranched_reactif_complex \\
\hline 168 & 48 & 82 & 44 & 85 & 40 & 80 & 44 & 84 & esp155 & $\leftrightarrow$ & esp147 & ESP168 & 3,4hexamerbranched_Intermediate \\
\hline 169 & 0 & 30 & 0 & 50 & 0 & 66 & 0 & 44 & esp $47+$ esp22 & $\leftrightarrow$ & esp156 + esp24 & ESP169 & (3)trimerisopentamer_reactif_complex \\
\hline 170 & 52 & 47 & 70 & 37 & 72 & 36 & 68 & 41 & esp156 & $\leftrightarrow$ & esp157 & ESP170 & (3)trimerisopentamer_Intermediate \\
\hline 171 & 48 & 82 & 44 & 85 & 40 & 80 & 44 & 84 & esp157 & $\leftrightarrow$ & esp150 & ESP171 & (3)trimerisopentamer... $\mathrm{H} 2 \mathrm{O}$ \\
\hline 172 & 0 & 13 & 0 & 18 & 0 & 20 & 0 & 21 & esp 84 & $\leftrightarrow$ & esp158 + esp24 & $\overline{\text { ESP172 }}$ & 4-ring2branched,3(trimer)branched_reactif_complex \\
\hline 173 & 28 & 16 & 28 & 15 & 29 & 17 & 25 & 18 & esp158 & $\leftrightarrow$ & esp159 & ESP173 & 4-ring2branched,3(trimer)branched_Intermediate \\
\hline 174 & 48 & 75 & 44 & 79 & 50 & 83 & 57 & 79 & esp159 & $\leftrightarrow$ & esp160 & ESP174 & 4-ring2branched,3(trimer)branched...H2O \\
\hline 175 & 0 & 30 & 0 & 50 & 0 & 66 & 0 & 44 & esp $84+$ esp 22 & $\leftrightarrow$ & esp161+esp24 & ESP175 & 4-ring2branched,3(trimer)branched_reactif_complex_V2 \\
\hline 176 & 52 & 47 & 70 & 37 & 72 & 36 & 68 & 41 & esp161 & $\leftrightarrow$ & esp162 & ESP176 & 4-ring2branched,3(trimer)branched_Intermediate_V2 \\
\hline 177 & 48 & 82 & 44 & 85 & 40 & 80 & 44 & 84 & esp162 & $\leftrightarrow$ & esp163 & ESP177 & 2,3dimerbranched4-ring_reactif_complex \\
\hline 178 & 0 & 21 & 0 & 14 & 0 & 30 & 0 & 10 & esp84 + esp22 & $\leftrightarrow$ & esp164 + esp24 & ESP178 & 2,3dimerbranched4-ring_Intermediate \\
\hline 179 & 47 & 33 & 45 & 23 & 48 & 24 & 43 & 28 & esp164 & $\leftrightarrow$ & esp165 & ESP179 & 2,3dimerbranched4-ring... $\mathrm{H} 2 \mathrm{O}$ \\
\hline 180 & 59 & 87 & 49 & 85 & 49 & 87 & 56 & 83 & esp165 & $\leftrightarrow$ & esp166 & ESP180 & 2,3dimerbranched4-ring_reactif_complex_V2 \\
\hline 181 & 0 & 21 & 0 & 14 & 0 & 30 & 0 & 10 & esp84 + esp22 & $\leftrightarrow$ & esp167 + esp24 & ESP181 & 2,3dimerbranched4-ring_Intermediate_v2 \\
\hline 182 & 47 & 33 & 45 & 23 & 48 & 24 & 43 & 28 & esp167 & $\leftrightarrow$ & esp168 & ESP182 & 2branched3trimerbranched4ring_reactif_complex \\
\hline 183 & 59 & 87 & 49 & 85 & 49 & 87 & 56 & 83 & esp168 & $\leftrightarrow$ & esp141 & ESP183 & 2branched3trimerbranched4ring_Intermediate \\
\hline 184 & 0 & 21 & 0 & 14 & 0 & 30 & 0 & 10 & esp84 + esp22 & $\leftrightarrow$ & esp169 + esp24 & ESP184 & 2branched3trimerbranched4ring... $\mathrm{H} 2 \mathrm{O}$ \\
\hline 185 & 47 & 33 & 45 & 23 & 48 & 24 & 43 & 28 & esp169 & $\leftrightarrow$ & esp170 & ESP185 & 1,2branched3dimer4-ring_reactif_complex \\
\hline 186 & 59 & 87 & 49 & 85 & 49 & 87 & 56 & 83 & esp170 & $\leftrightarrow$ & esp171 & ESP186 & 1,2branched3dimer4-ring_Intermediate \\
\hline 187 & 0 & 21 & 0 & 14 & 0 & 30 & 0 & 10 & esp $160+$ esp22 & $\leftrightarrow$ & esp $172+$ esp24 & ESP187 & 1,2branched3dimer4-ring... $\mathrm{H} 2 \mathrm{O}$ \\
\hline 188 & 47 & 33 & 45 & 23 & 48 & 24 & 43 & 28 & esp172 & $\leftrightarrow$ & esp173 & ESP188 & 2,4branched3dimer4-ring_reactif_complex \\
\hline 189 & 59 & 87 & 49 & 85 & 49 & 87 & 56 & 83 & esp 173 & $\leftrightarrow$ & esp174 & ESP189 & 2,4branched3dimer4-ring_Intermediate \\
\hline
\end{tabular}




\begin{tabular}{|c|c|c|c|c|c|c|c|c|c|c|c|c|c|}
\hline 190 & 0 & 13 & 0 & 18 & 0 & 20 & 0 & 21 & \multirow{3}{*}{$\begin{array}{l}\text { esp166 } \\
\text { esp175 } \\
\text { esp176 } \\
\end{array}$} & $\leftrightarrow$ & \multirow{3}{*}{$\begin{array}{c}\text { esp175 + esp24 } \\
\text { esp176 } \\
\text { esp174 }\end{array}$} & \multirow{3}{*}{$\begin{array}{l}\text { ESP190 } \\
\text { ESP191 } \\
\text { ESP192 } \\
\end{array}$} & 2,4branched3dimer4-ring... $\mathrm{H} 2 \mathrm{O}$ \\
\hline 191 & 28 & 16 & 28 & 15 & 29 & 17 & 25 & 18 & & $\leftrightarrow$ & & & 4-ring2trimer4branched_reactif_complex \\
\hline 192 & 48 & 75 & 44 & 79 & 50 & 83 & 57 & 79 & & $\leftrightarrow$ & & & 4-ring2trimer4branched_Intermediate \\
\hline 193 & 0 & 21 & 0 & 14 & 0 & 30 & 0 & 10 & \multirow{3}{*}{$\begin{array}{c}\text { esp160 + esp22 } \\
\text { esp177 } \\
\text { esp178 }\end{array}$} & $\leftrightarrow$ & \multirow{3}{*}{$\begin{array}{c}\text { esp177 + esp24 } \\
\text { esp178 } \\
\text { esp179 } \\
\end{array}$} & \multirow{3}{*}{$\begin{array}{l}\text { ESP193 } \\
\text { ESP194 } \\
\text { ESP195 } \\
\end{array}$} & 4-ring2trimer4branched... $\mathrm{H} 2 \mathrm{O}$ \\
\hline 194 & 47 & 33 & 45 & 23 & 48 & 24 & 43 & 28 & & $\leftrightarrow$ & & & 4-ring2tetramerbranched_reactif_complex \\
\hline 195 & 59 & 87 & 49 & 85 & 49 & 87 & 56 & 83 & & $\leftrightarrow$ & & & 4-ring2tetramerbranched_Intermediate \\
\hline 196 & 0 & 13 & 0 & 18 & 0 & 20 & 0 & 21 & \multirow{3}{*}{$\begin{array}{l}\text { esp169 } \\
\text { esp180 } \\
\text { esp181 } \\
\end{array}$} & $\leftrightarrow$ & \multirow{3}{*}{$\begin{array}{c}\text { esp180 + esp24 } \\
\text { esp181 } \\
\text { esp179 }\end{array}$} & \multirow{3}{*}{$\begin{array}{l}\text { ESP196 } \\
\text { ESP197 } \\
\text { ESP198 } \\
\end{array}$} & 4-ring2tetramerbranched... $\mathrm{H} 2 \mathrm{O}$ \\
\hline 197 & 28 & 16 & 28 & 15 & 29 & 17 & 25 & 18 & & $\leftrightarrow$ & & & 4-ring2trimer3branched_reactif_complex \\
\hline 198 & 48 & 75 & 44 & 79 & 50 & 83 & 57 & 79 & & $\leftrightarrow$ & & & 4-ring2trimer3branched_Intermediate \\
\hline 199 & 0 & 30 & 0 & 50 & 0 & 66 & 0 & 44 & \multirow{3}{*}{$\begin{array}{c}\text { esp160 + esp22 } \\
\text { esp182 } \\
\text { esp183 }\end{array}$} & $\leftrightarrow$ & \multirow{3}{*}{$\begin{array}{c}\text { esp182 + esp24 } \\
\text { esp183 } \\
\text { esp184 } \\
\end{array}$} & \multirow{3}{*}{$\begin{array}{l}\text { ESP199 } \\
\text { ESP200 } \\
\text { ESP201 }\end{array}$} & 4-ring2trimer3branched... $\mathrm{H} 2 \mathrm{O}$ \\
\hline 200 & 52 & 47 & 70 & 37 & 72 & 36 & 68 & 41 & & $\leftrightarrow$ & & & 4-ring2tetramer_reactif_complex \\
\hline 201 & 48 & 82 & 44 & 85 & 40 & 80 & 44 & 84 & & $\leftrightarrow$ & & & 4-ring2tetramer_Intermediate \\
\hline 202 & 0 & 30 & 0 & 50 & 0 & 66 & 0 & 44 & \multirow{3}{*}{$\begin{array}{c}\text { esp160 + esp22 } \\
\text { esp185 } \\
\text { esp186 }\end{array}$} & $\leftrightarrow$ & \multirow{3}{*}{$\begin{array}{c}\text { esp185 + esp24 } \\
\text { esp186 } \\
\text { esp187 }\end{array}$} & \multirow{3}{*}{$\begin{array}{l}\text { ESP202 } \\
\text { ESP203 } \\
\text { ESP204 } \\
\end{array}$} & 4-ring2tetramer... $\mathrm{H} 2 \mathrm{O}$ \\
\hline 203 & 52 & 47 & 70 & 37 & 72 & 36 & 68 & 41 & & $\leftrightarrow$ & & & 4-ring2tetramer_reactif_complex_V2 \\
\hline 204 & 48 & 82 & 44 & 85 & 40 & 80 & 44 & 84 & & $\leftrightarrow$ & & & 4-ring2tetramer_Intermediate_V2 \\
\hline 205 & 0 & 30 & 0 & 50 & 0 & 66 & 0 & 44 & \multirow{3}{*}{$\begin{array}{c}\text { esp160 + esp22 } \\
\text { esp188 } \\
\text { esp189 }\end{array}$} & $\leftrightarrow$ & \multirow{3}{*}{$\begin{array}{c}\text { esp188 + esp24 } \\
\text { esp189 } \\
\text { esp190 }\end{array}$} & \multirow{3}{*}{$\begin{array}{l}\text { ESP205 } \\
\text { ESP206 } \\
\text { ESP207 } \\
\end{array}$} & 2,2bishexamerbranched_reactif_complex \\
\hline 206 & 52 & 47 & 70 & 37 & 72 & 36 & 68 & 41 & & $\leftrightarrow$ & & & 2,2bishexamerbranched_Intermediate \\
\hline 207 & 48 & 82 & 44 & 85 & 40 & 80 & 44 & 84 & & $\leftrightarrow$ & & & 2,2bishexamerbranched... $\mathrm{H} 2 \mathrm{O}$ \\
\hline 208 & 0 & 30 & 0 & 50 & 0 & 66 & 0 & 44 & & $\leftrightarrow$ & esp191 + esp24 & ESP208 & 2,2,3pentamerbranched_reactif_complex \\
\hline 209 & 52 & 47 & 70 & 37 & 72 & 36 & 68 & 41 & esp191 & $\leftrightarrow$ & esp192 & ESP209 & 2,2,3pentamerbranched_Intermediate \\
\hline 210 & 48 & 82 & 44 & 85 & 40 & 80 & 44 & 84 & esp192 & $\leftrightarrow$ & esp193 & ESP210 & 2,2,3pentamerbranched...H2O \\
\hline 211 & 0 & 0 & 0 & 0 & 0 & 0 & 0 & 0 & esp111 + esp22 & $\leftrightarrow$ & esp194 + esp24 & ESP211 & 2,3,3pentamerbranched_reactif_complex \\
\hline 212 & 46 & 31 & 52 & 42 & 48 & 43 & 54 & 39 & esp194 & $\leftrightarrow$ & esp195 & ESP212 & 2,3,3pentamerbranched_Intermediate \\
\hline 213 & 25 & 77 & 13 & 63 & 18 & 46 & 35 & 71 & esp195 & $\leftrightarrow$ & esp196 & ESP213 & 2,3,3pentamerbranched...H2O \\
\hline 214 & 0 & 30 & 0 & 50 & 0 & 66 & 0 & 44 & esp111+esp22 & $\leftrightarrow$ & esp197+ esp24 & ESP214 & 2,2branched3(dimer)branched4-ring_reactif_complex \\
\hline 215 & 52 & 47 & 70 & 37 & 72 & 36 & 68 & 41 & esp197 & $\leftrightarrow$ & esp198 & ESP215 & 2,2branched3(dimer)branched4-ring_Intermediate \\
\hline 216 & 48 & 82 & 44 & 85 & 40 & 80 & 44 & 84 & esp198 & $\leftrightarrow$ & esp199 & ESP216 & 2,2branched3(dimer)branched4-ring... $\mathrm{H} 2 \mathrm{O}$ \\
\hline 217 & 0 & 21 & 0 & 14 & 0 & 30 & 0 & 10 & esp111 + esp22 & $\leftrightarrow$ & esp200+ esp24 & ESP217 & 2,3branched3(dimer)branched4-ring_reactif_complex \\
\hline 218 & 47 & 33 & 45 & 23 & 48 & 24 & 43 & 28 & esp200 & $\leftrightarrow$ & esp201 & ESP218 & 2,3branched3(dimer)branched4-ring_Intermediate \\
\hline 219 & 59 & 87 & 49 & 85 & 49 & 87 & 56 & 83 & esp201 & $\leftrightarrow$ & esp202 & ESP219 & 2,3branched3(dimer)branched4-ring....H2O \\
\hline 220 & 0 & 21 & 0 & 14 & 0 & 30 & 0 & 10 & esp169 & $\leftrightarrow$ & esp203 + esp24 & ESP220 & 4-ring2branched_reactif_complex \\
\hline 221 & 47 & 33 & 45 & 23 & 48 & 24 & 43 & 28 & esp203 & $\leftrightarrow$ & esp204 & ESP221 & 4-ring2branched_Intermediate \\
\hline 222 & 59 & 87 & 49 & 85 & 49 & 87 & 56 & 83 & esp204 & $\leftrightarrow$ & esp202 & ESP222 & 4-ring2branched...H2O \\
\hline 223 & 0 & 0 & 0 & 0 & 0 & 0 & 0 & 0 & esp101+ esp22 & $\leftrightarrow$ & esp205 + esp24 & ESP223 & 4-ring2branched_reactif_complex_V2 \\
\hline 224 & 46 & 31 & 52 & 42 & 48 & 43 & 54 & 39 & esp205 & $\leftrightarrow$ & esp206 & ESP224 & 4-ring2branched_Intermediate_V2 \\
\hline 225 & 25 & 77 & 13 & 63 & 18 & 46 & 35 & 71 & esp206 & $\leftrightarrow$ & esp207 & ESP225 & 4-ring2branched_reactif_complex_V3 \\
\hline 226 & 0 & 0 & 0 & 0 & 0 & 0 & 0 & 0 & esp84 + esp22 & $\leftrightarrow$ & esp208 + esp24 & ESP226 & 4-ring2branched_Intermediate_V3 \\
\hline 227 & 46 & 31 & 52 & 42 & 48 & 43 & 54 & 39 & esp208 & $\leftrightarrow$ & esp209 & ESP227 & 2,4bis4-ringbranched_reactif_complex \\
\hline 228 & 25 & 77 & 13 & 63 & 18 & 46 & 35 & 71 & esp209 & $\leftrightarrow$ & esp210 & ESP228 & 2,4bis4-ringbranched_Intermediate \\
\hline 229 & 0 & 0 & 0 & 0 & 0 & 0 & 0 & 0 & esp84 + esp22 & $\leftrightarrow$ & esp211 + esp24 & ESP229 & 2,4bis4-ringbranched...H2O \\
\hline 230 & 46 & 31 & 52 & 42 & 48 & 43 & 54 & 39 & esp211 & $\leftrightarrow$ & esp212 & ESP230 & 2(Dimer)4-ringbranched_reactif_complex \\
\hline 231 & 25 & 77 & 13 & 63 & 18 & 46 & 35 & 71 & esp212 & $\leftrightarrow$ & esp213 & ESP231 & 2(Dimer)4-ringbranched_Intermediate \\
\hline 232 & 0 & 0 & 0 & 0 & 0 & 0 & 0 & 0 & esp160+esp22 & $\leftrightarrow$ & esp214 + esp24 & ESP232 & 2(Dimer)4-ringbranched_Intermediate... $\mathrm{H} 2 \mathrm{O}$ \\
\hline 233 & 46 & 31 & 52 & 42 & 48 & 43 & 54 & 39 & esp214 & $\leftrightarrow$ & esp215 & ESP233 & 1,2-4-ringbranched_reactif_complex \\
\hline 234 & 25 & 77 & 13 & 63 & 18 & 46 & 35 & 71 & esp215 & $\leftrightarrow$ & esp216 & ESP234 & 1,2-4-ringbranched_Intermediate \\
\hline 235 & 0 & 0 & 0 & 0 & 0 & 0 & 0 & 0 & esp160 + esp22 & $\leftrightarrow$ & esp217 + esp24 & ESP235 & 1,2-4-ringbranched... $\mathrm{H} 2 \mathrm{O}$ \\
\hline 236 & 46 & 31 & 52 & 42 & 48 & 43 & 54 & 39 & esp217 & $\leftrightarrow$ & esp218 & ESP236 & 2,4-4-ringbranched_reactif_complex \\
\hline 237 & 25 & 77 & 13 & 63 & 18 & 46 & 35 & 71 & esp218 & $\leftrightarrow$ & esp219 & ESP237 & 2,4-4-ringbranched_Intermediate \\
\hline
\end{tabular}




\begin{tabular}{|c|c|c|c|c|c|c|c|c|c|c|c|c|c|}
\hline 238 & 0 & 30 & 0 & 50 & 0 & 66 & 0 & 44 & \multirow{3}{*}{$\begin{array}{c}\text { esp18 + esp22 } \\
\text { esp220 } \\
\text { esp221 }\end{array}$} & $\leftrightarrow$ & \multirow{3}{*}{$\begin{array}{c}\text { esp220 + esp24 } \\
\text { esp221 } \\
\text { esp222 }\end{array}$} & \multirow{3}{*}{$\begin{array}{l}\text { ESP238 } \\
\text { ESP239 } \\
\text { ESP240 }\end{array}$} & 2,4-4-ringbranched... $\mathrm{H} 2 \mathrm{O}$ \\
\hline 239 & 52 & 47 & 70 & 37 & 72 & 36 & 68 & 41 & & $\leftrightarrow$ & & & Isoheptamerbranched_reactif_complex_V2 \\
\hline 240 & 48 & 82 & 44 & 85 & 40 & 80 & 44 & 84 & & $\leftrightarrow$ & & & Isoheptamerbranched_Intermediate_V2 \\
\hline 241 & 0 & 13 & 0 & 18 & 0 & 20 & 0 & 21 & esp62 & $\leftrightarrow$ & esp223 + esp24 & ESP241 & H2O_bis \\
\hline 242 & 28 & 16 & 28 & 15 & 29 & 17 & 25 & 18 & esp223 & $\leftrightarrow$ & esp224 & ESP242 & Isopentamer_reactif_complex \\
\hline 243 & 48 & 75 & 44 & 79 & 50 & 83 & 57 & 79 & esp224 & $\leftrightarrow$ & esp222 & & \\
\hline 244 & 0 & 13 & 0 & 18 & 0 & 20 & 0 & 21 & esp 30 & $\leftrightarrow$ & esp225 + esp24 & & \\
\hline 245 & 28 & 16 & 28 & 15 & 29 & 17 & 25 & 18 & esp225 & $\leftrightarrow$ & esp226 & & \\
\hline 246 & 48 & 75 & 44 & 79 & 50 & 83 & 57 & 79 & esp226 & $\leftrightarrow$ & esp222 & & \\
\hline 247 & 0 & 0 & 0 & 0 & 0 & 0 & 0 & 0 & esp222 + esp22 & $\leftrightarrow$ & esp227 + esp24 & & \\
\hline 248 & 46 & 31 & 52 & 42 & 48 & 43 & 54 & 39 & esp227 & $\leftrightarrow$ & esp228 & & \\
\hline 249 & 25 & 77 & 13 & 63 & 18 & 46 & 35 & 71 & esp228 & $\leftrightarrow$ & esp229 & & \\
\hline 250 & 0 & 21 & 0 & 14 & 0 & 30 & 0 & 10 & esp222 + esp22 & $\leftrightarrow$ & esp230 + esp24 & & \\
\hline 251 & 47 & 33 & 45 & 23 & 48 & 24 & 43 & 28 & esp230 & $\leftrightarrow$ & esp231 & & \\
\hline 252 & 59 & 87 & 49 & 85 & 49 & 87 & 56 & 83 & esp231 & $\leftrightarrow$ & esp232 & & \\
\hline 253 & 0 & 30 & 0 & 50 & 0 & 66 & 0 & 44 & esp222 + esp22 & $\leftrightarrow$ & esp233 + esp24 & & \\
\hline 254 & 52 & 47 & 70 & 37 & 72 & 36 & 68 & 41 & esp233 & $\leftrightarrow$ & esp234 & & \\
\hline 255 & 48 & 82 & 44 & 85 & 40 & 80 & 44 & 84 & esp234 & $\leftrightarrow$ & esp235 & & \\
\hline 256 & 0 & 30 & 0 & 50 & 0 & 66 & 0 & 44 & esp222 + esp22 & $\leftrightarrow$ & esp236+ esp24 & & \\
\hline 257 & 52 & 47 & 70 & 37 & 72 & 36 & 68 & 41 & esp236 & $\leftrightarrow$ & esp237 & & \\
\hline 258 & 48 & 82 & 44 & 85 & 40 & 80 & 44 & 84 & esp237 & $\leftrightarrow$ & esp238 & & \\
\hline
\end{tabular}

Table S2: Complete list of equilibrium reactions for extended reaction pathway (until $8 \mathrm{Si}$ atoms). First column corresponds to the ID number of each reaction considered. Columns two to nine corresponds to the activation barriers (in kJ.mol-1) of forward and backward reactions for no-templates and $\mathrm{TMA}^{+}, \mathrm{TEA}^{+}$and TPA ${ }^{+}$templates. Columns ten to twelve corresponds to the details of the reactions including the id of species as used in SPPARKS code. Columns thirteen and fourteen corresponds to the ID code and name of the species considered in the study. 
(a)
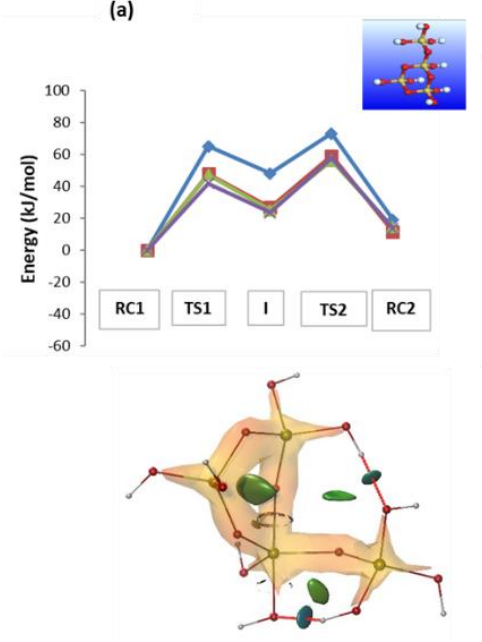

(b)
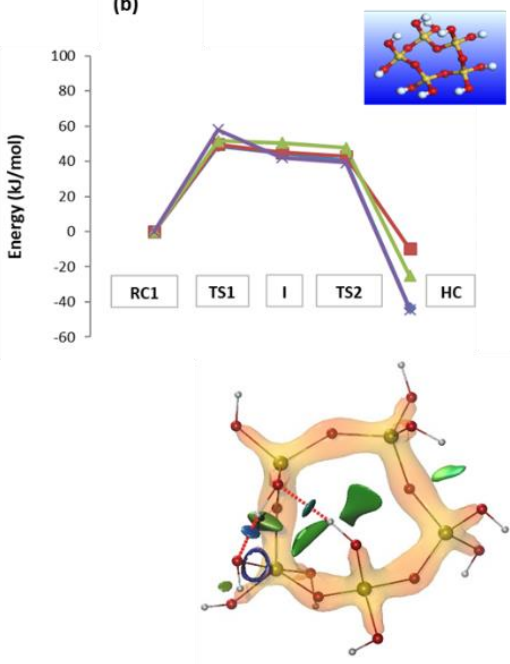

(c)
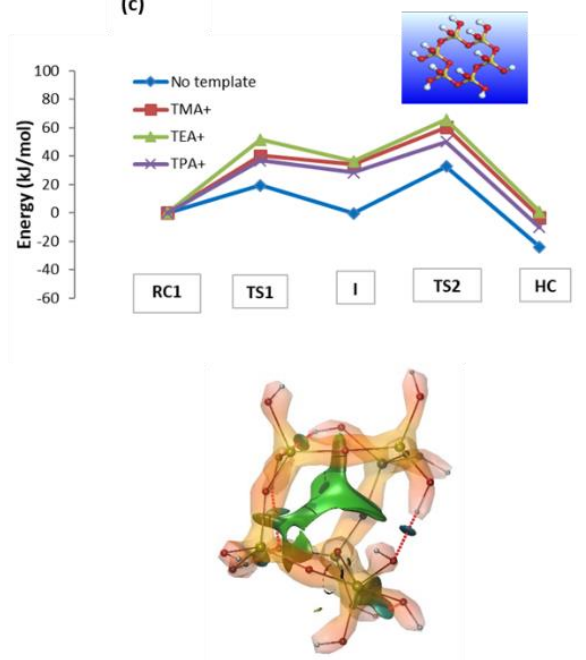

Figure S5: Energy barriers profiles and $\mathrm{NCl}$ Analysis (Intermediate state) for cyclic oligomerisation process (Scheme 1) without and with templates (TEA+, TMA+ and TPA+). Colours represents different type of non-covalent interactions: $\mathrm{H}$-bonds (blue); vdW (green). We observe that the release water mechanism is fully dependent of cyclic oligomers size. It is thus difficult to predict the energy barriers with BEP models for cyclic species.

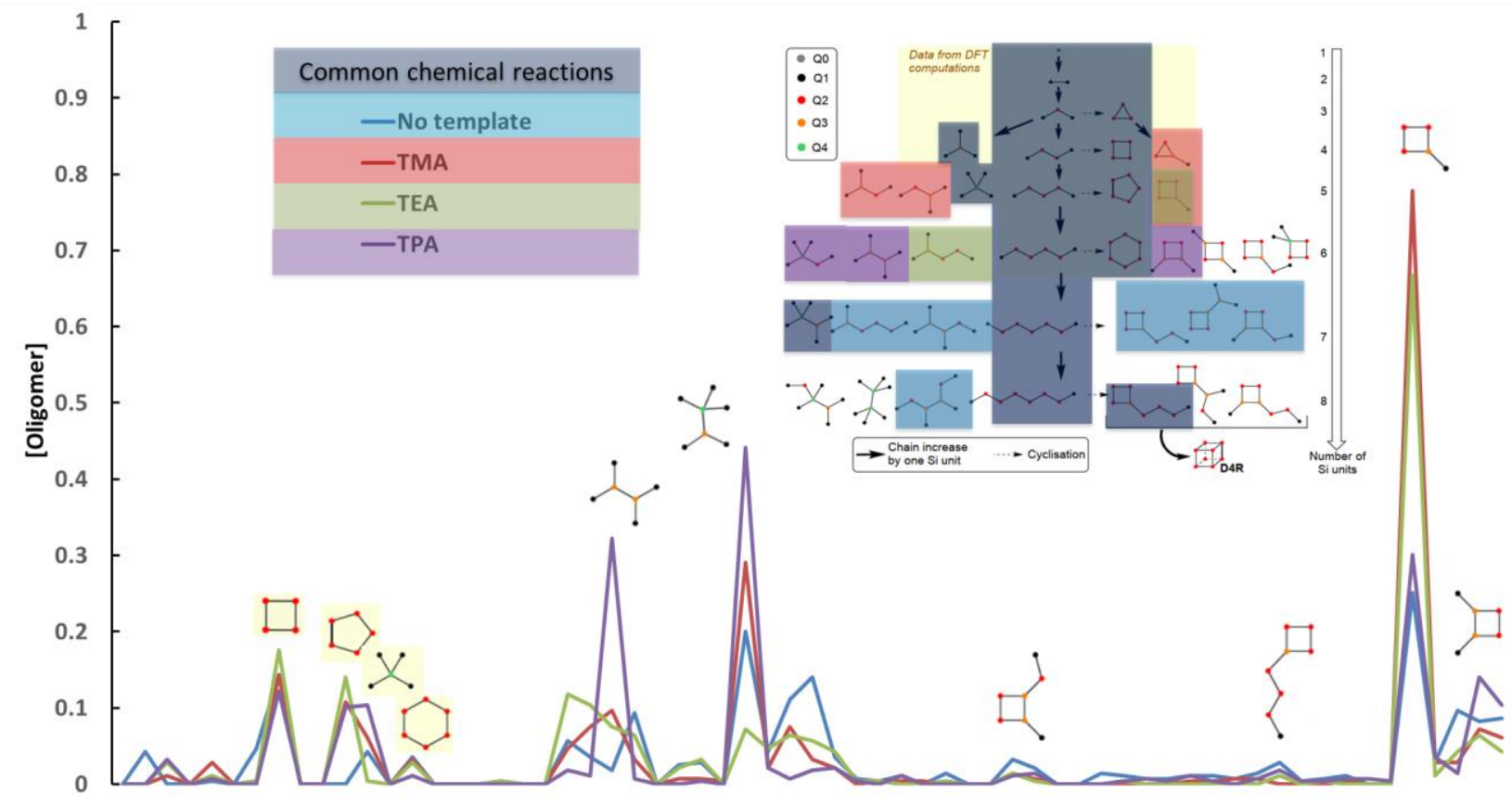

Figure S6: Steady state concentrations (in $\mathrm{mmol}^{-1} \mathrm{~L}^{-1}$ ) of major chemical species calculated by $\mathrm{kMC}$ with the full-extended chemical reaction pathway (Scheme 2). In the top right: an extract of the fullextended chemical reaction pathway with the effect of the different templates on the reactions. In black colour: the common chemical reactions shared by all templates, blue colour: no template specific reactions, red colour: TMA specific reactions, green colour: TEA specific reactions, purple colour: TPA specific reactions. 

Bibliographie:

[1] Julia Contreras-García, Erin R. Johnson, Shahar Keinan, Robin Chaudret, Jean-Philip Piquemal, David N. Beratan, Weitao Yang, J. Chem. Theory Comput. 2011, 7, 3, 625-632

[2] Erin R. Johnson, Shahar Keinan, Paula Mori-Sánchez, Julia Contreras-García, Aron J. Cohen, Weitao Yang, J. Am. Chem. Soc. 2010, 132, 18, 6498-6506 